\title{
Performance of the SUBSTOR-potato model across contrasting growing conditions
}

Rubi Raymundo ${ }^{\mathrm{a}, *}$

r.raymundo@ufl.edu

Senthold Assenga

Rishi Prassad ${ }^{a}$

Ulrich Kleinwechter ${ }^{b}$

Juan Concha ${ }^{c}$

Bruno Condoric

Walter Bowen

Joost Wolf ${ }^{\mathrm{d}}$

Jørgen E. Olesen

Qiaoxue Dong ${ }^{f}$

Lincoln Zotarellia

Manuel Gastelo ${ }^{c}$

Ashok Alva ${ }^{g}$

Maria Travasso ${ }^{h}$

Roberto Quiroz

Vijay Arora'

Wendy Graham ${ }^{a}$

Cheryl Porter

aAgriculture and Biological toridaEngineering Department, University of Florida, Gainesville, FL, USA

${ }^{b}$ International Institute for Applied Systems Analysis (IIASA), Laxenburg, Austria

'International Potato Center (CIP), Lima, Peru

dPlant Production Systems Group, Wageningen University, The Netherlands

eDepartment of Agroecology, Aarhus University, Tjele, Denmark

${ }^{\mathrm{f} C h i n a ~ A g r i c u l t u r a l ~ U n i v e r s i t y, ~ B e i j i n g, ~ C h i n a ~}$

9United States Department of Agriculture-Agricultural Research Service (USDA-ARS), Sidney, MT, USA 


\section{Abstract}

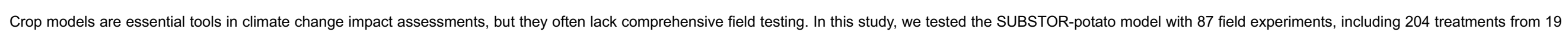

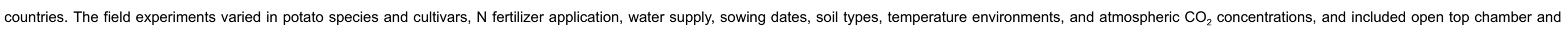

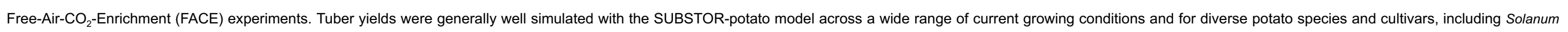

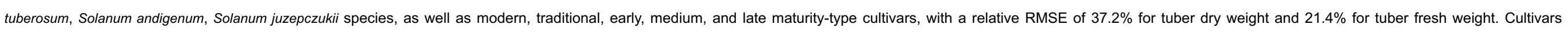

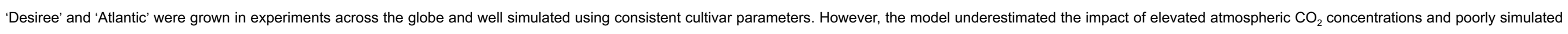

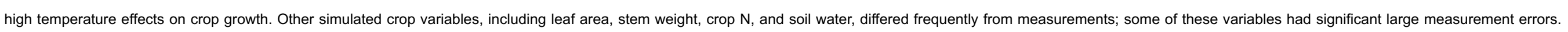

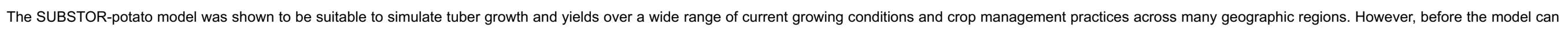
be used effectively in climate change impact assessments, it requires improved model routines to capture the impacts of elevated atmospheric $\mathrm{CO}_{2}$ and high temperatures on crop growth.

\section{Keywords: SUBSTOR-potato; Potato; Crop modeling; Model performance; $\mathrm{CO}_{2} ;$ High temperature}

\section{Introduction}

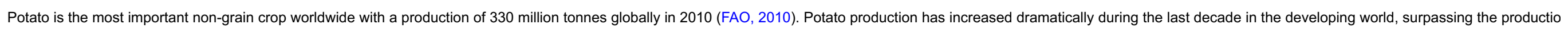

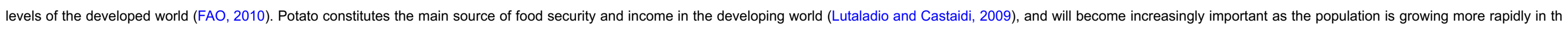

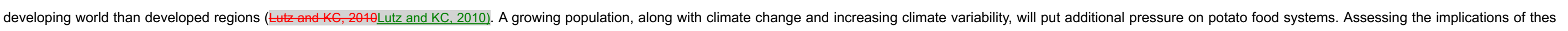
trends requires integrating crop models when evaluating the impact of new technologies and strategies for adapting to climate change.

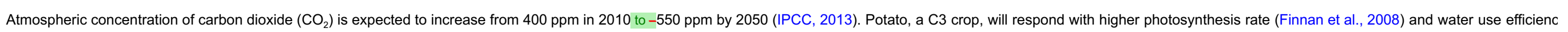

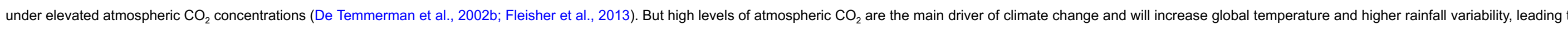

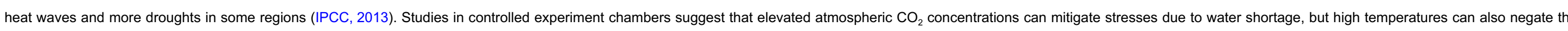
positive effects of increased atmospheric $\mathrm{CO}_{2}$ concentrations on crop production (Kaminski et al., 2014).

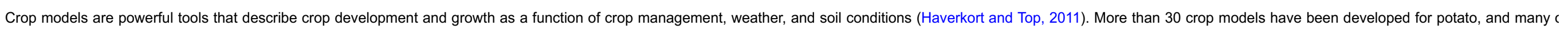

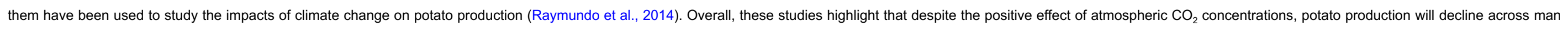

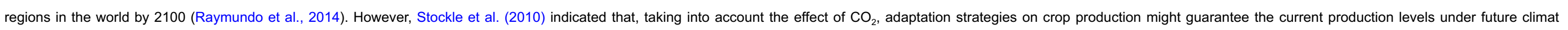

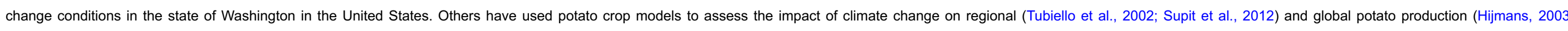

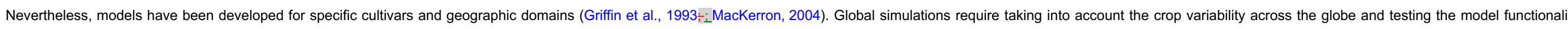

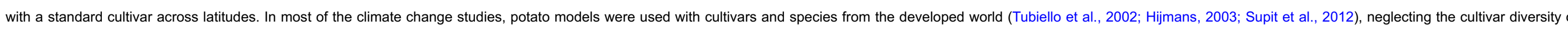

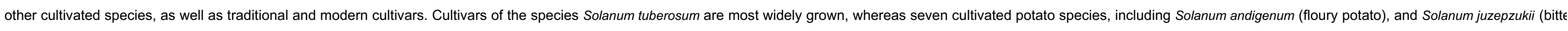
potato), coexist in the tropical Andes (Huaman and Spooner, 2002). Also, several hybrids of various species are grown in the developing world (Thiele et al., 2007), where the use of potato models is limited. 


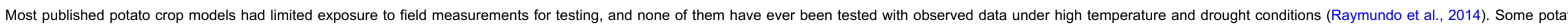

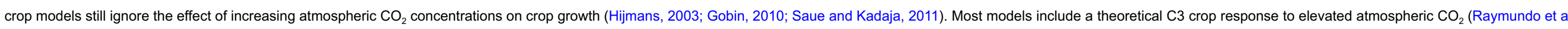

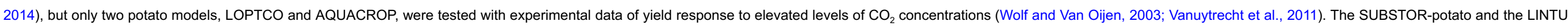

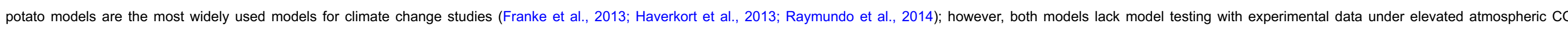

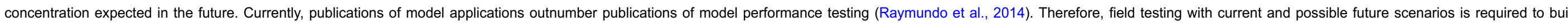

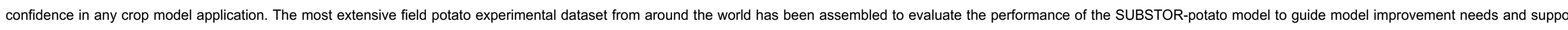
future model applications.

\section{Material and methods}

\subsection{The model}

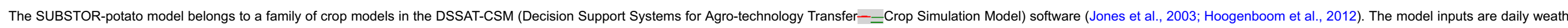

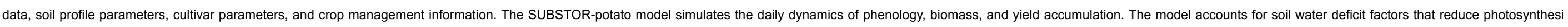

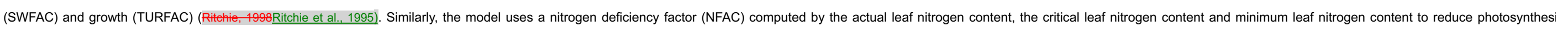

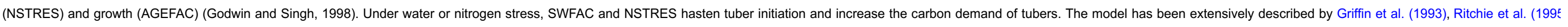
and Singh et al. (1998). Following is a brief summary of the model.

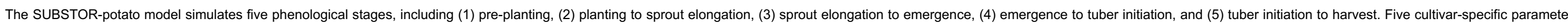

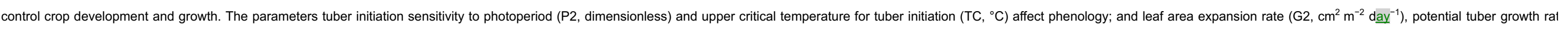
(G3, $\left.\mathrm{gm}^{-2} \mathrm{day}^{-1}\right)$, and an index that suppresses tuber growth (PD, dimensionless) affect biomass accumulation (Griffin et al., 1993).

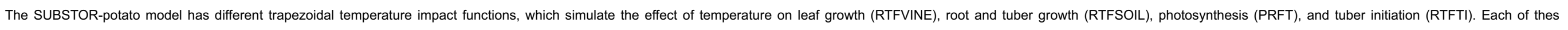

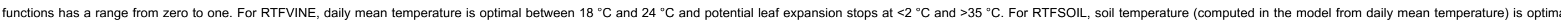

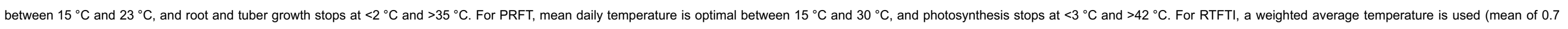

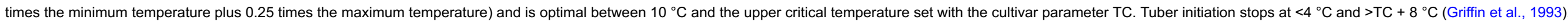

\subsubsection{Tuber initiation}

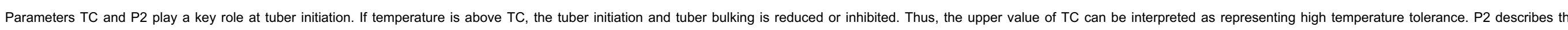

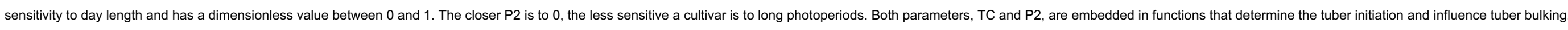

The relative temperature function for tuber initiation (RTFTI) is described as follows:

RTFTI $=0$; (TEMP $<=4)$

RTFTI $=1-(1 / 36)^{*}(10-$ TEMP) $;($ TEMP $>4$ \& TEMP $<=10)$

RTFTI $=1 ;($ TEMP $>10$ \& TEMP $<=$ TC $)$

Please align formula to leftRTFTI $=1-(1 / 64)^{*}(\text { TEMP-TC })^{2} ;($ TEMP $>$ TC \& TEMP $<=$ TC + 8)

Here, RTFTI is a function of weighted average temperature $\left(T E M P=\operatorname{tmin}^{*} 0.75+\operatorname{tmax} x^{*} 0.25\right)$ and critical temperature (TC).

The relative daylength function for tuber initiation (RDLFTI) is described as follows:

RDLFTI $=(1-P 2)+0.00694^{*} 2^{*}(24-P H P E R)^{2}$ 
Here, RDLFTI is a function of daylength in hours (PHPER) and sensitivity to daylength (P2). RDLFTI equals 1 when photoperiod is less than 12 hours.

\subsubsection{Biomass accumulation after tuber initiation and partitioning}

In the SUBSTOR-potato model, $\mathrm{CO}_{2}$ concentrations affect the daily potential carbon fixation and potential tuber growth. The potential carbon fixation rate is described as follows:

PCARB $=$ RUE ${ }^{*}$ PAR/PLANTS ${ }^{*}\left(1-\operatorname{EXP}\left(-0.55^{*}{ }^{*}\right.\right.$ AI $){ }^{*}{ }^{*} \mathrm{CO}_{2}$

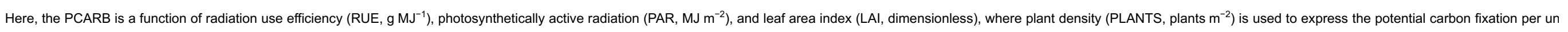

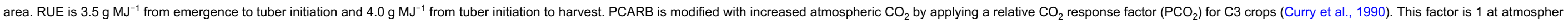

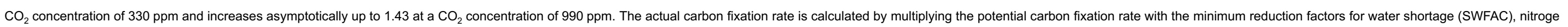
stress (NSTRES), or temperature factor that affects photosynthesis (PRFT) (Griffin et al., 1993).

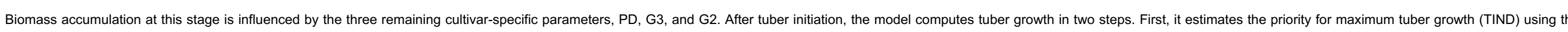
sink strength (DTII) and the carbon demand of tubers after tuber initiation (DEVEFF):

TIND $=$ DTIlavg ${ }^{*}(1 / N F A C)^{*}$ DEVEFF; NFAC $>1$

TIND = DTIlavg*DEVEFF; NFAC $<1$

DTII = RTFTI; if no stress

$\mathrm{DTII}=\mathrm{RTFTI}+0.5^{*}(1-\min (\mathrm{SWFAC}, \mathrm{NSTRES}, 1))$

DEVEFF $=\min \left((X S T A G E-2) * 10^{*} P D, 1\right)$

XSTAGE $=2.0+($ CUMRTFVINE $) / 100$

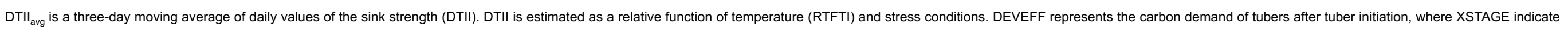

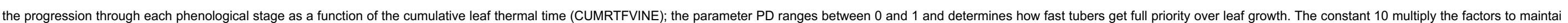
the result between 0 and 1 .

Second, the model estimates the potential tuber growth (PTUBGR, g plant ${ }^{-1}$ day $^{-1}$ ) as a function of potential tuber growth rate (G3), relative temperature factor for root growth (RTFSOIL), and plant density (PLANTS, plants $\mathrm{m}^{-2}$ ): PTUBGR $=\mathrm{G} 3{ }^{*} \mathrm{PCO}_{\mathbf{2 2}}{ }^{*}{ }^{*}$ RTFSOIL/PLANTS

Actual tuber growth (GROTUB, g plant ${ }^{-1}$ day $^{-1}$ ) is a function of potential tuber growth affected by TIND, and water and nitrogen shortages: GROTUB $=$ PTUBGR* $m$ in $(\text { TURFAC,AGEFAC, } 1)^{*}$ TIND

Actual leaf expansion (PLAG) is a function of potential leaf expansion (G2), limited by temperature (RTFVINE), water (TURFAC) and nitrogen (AGEFAC) shortages: PLAG $=$ G2*RTFVINE/PLANTS * $\min ($ TURFAC,AGEFAC, 1

Leaf (GROLF), stem (GROSTM) and root (GRORT) growth are computed as follows:

GROLF = PLAG/LALWR

GROSTM $=$ GROLF $^{*} 0.75$

GRORT $=(\text { GROLF }+ \text { GROSTM })^{*} 0.2$

where the leaf area to leaf weight ratio (LALWR, $270 \mathrm{~cm} \mathrm{~g}^{-1}$ ) is a constant through the crop development. 


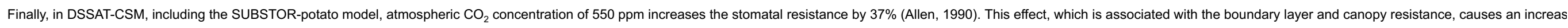
in transpiration efficiency.

\section{Experimental data}

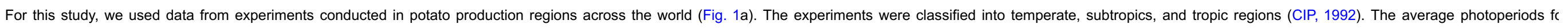

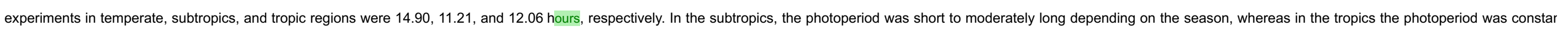

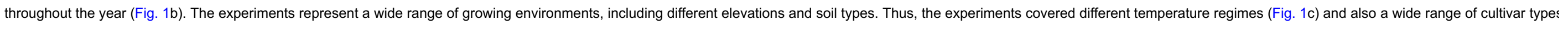

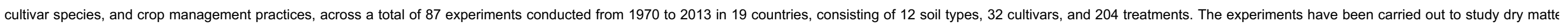

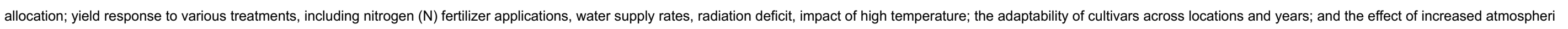

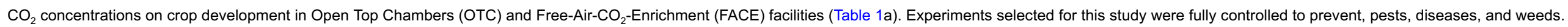
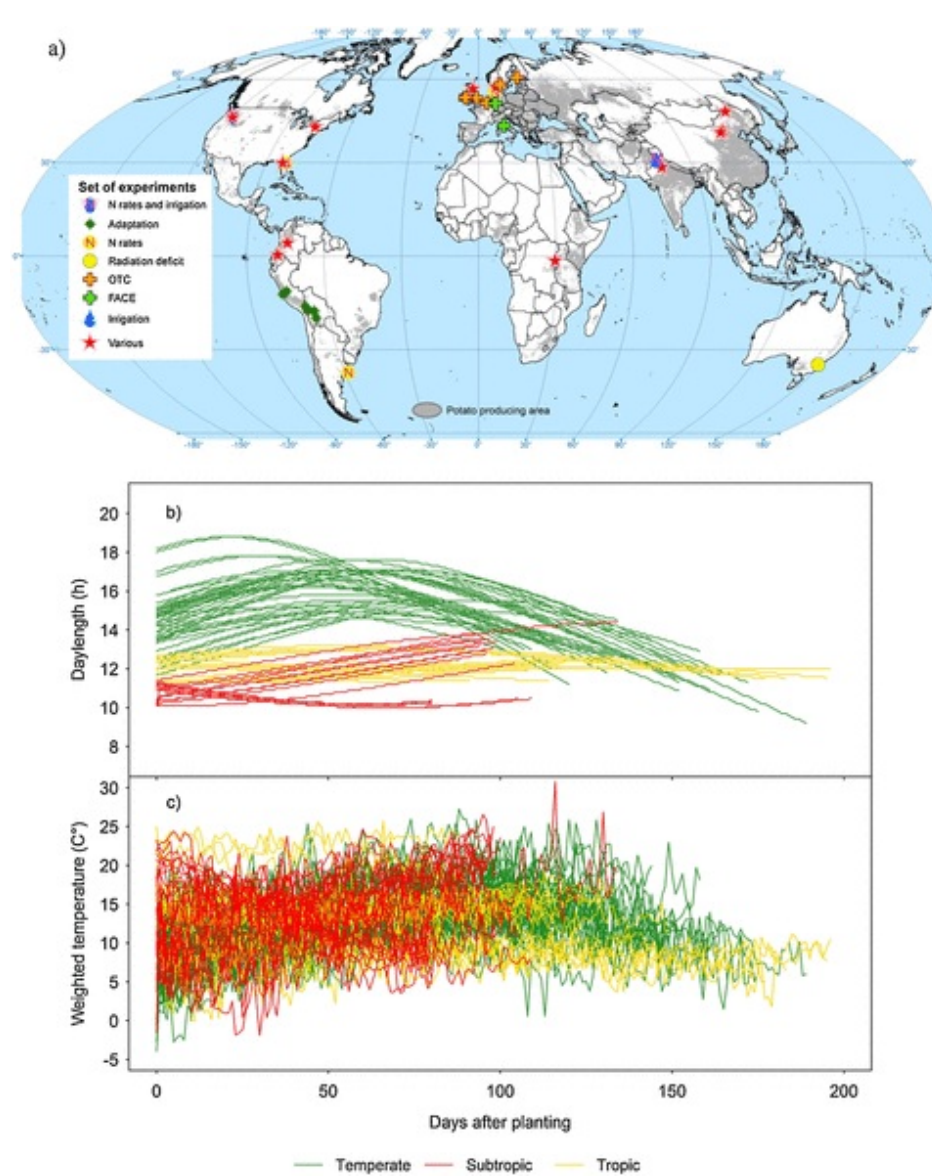

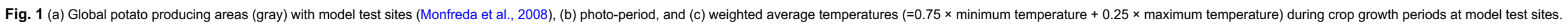
alt-text: Fig. 1 


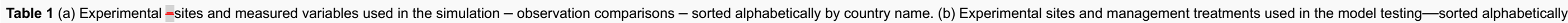
by country name.

\begin{tabular}{|c|c|c|c|c|c|c|c|c|c|c|c|c|}
\hline $\begin{array}{c}\text { (A) } \\
\text { Location }\end{array}$ & Year & lat $^{\mathrm{a}}$ & $\operatorname{lon}^{\mathrm{b}}$ & alt $^{\mathrm{c}}$ & Objective $^{d}$ & $\operatorname{tr}^{\mathrm{e}}$ & cult $^{f}$ & rep $^{9}$ & In-season sampling & Measured variables ${ }^{h}$ & Soil Texture' & Reference \\
\hline Argentina, Balcarce & 1991 & -37.8 & -58.3 & 97 & $\mathrm{~N}$ rates & 4 & 1 & & 5 & tu & $\mathrm{CL}$ & Travasso et al. (1996) \\
\hline Australia & 1970 & -35.0 & 149.0 & & Radiation deficit & 3 & 1 & & 10 & tuf,LAl & SC & Hoogenboom et al. (2012) \\
\hline Belgium, Tervuren & 1998-1999 & 50.8 & 4.5 & 97 & $\mathrm{CO}_{2} \mathrm{OTC}$ & 1 & 1 & $3-6$ & 2 & tu,le,st,to,LAI & SiL & De Temmerman et al. (2002a) \\
\hline Bolivia, Belen & 1997 & -16.0 & -68.7 & 3640 & Cultivar adaptation & 1 & 3 & 3 & 3 & tu,le & $\mathrm{CL}$ & Condori et al. (2010) \\
\hline Bolivia, Chinoli & 1997 & -19.6 & -65.3 & 3450 & Cultivar adaptation & 1 & 1 & 3 & 4 & tu,le,st,ro,to & SL & Condori et al. (2010) \\
\hline Bolivia, Koari & 1997 & -17.4 & -65.6 & 3500 & Cultivar adaptation & 1 & 3 & 3 & 3 & tu,le,st & $\mathrm{CL}$ & Condori et al. (2010) \\
\hline Bolivia, Patacamaya & 1997 & -17.2 & -68.0 & 3780 & Cultivar adaptation & 1 & 2 & & $3-4$ & tu,le,st,ro & $\mathrm{SiCl}$ & Condori et al. (2010) \\
\hline Bolivia, Patacamaya & $1998(2)$ & -17.2 & -68.0 & 3780 & Cultivar adaptation & 1 & 2 & 3 & $3-4$ & tu,le,st,ro & $\mathrm{SCL}$ & Condori et al. (2010) \\
\hline Bolivia, Toralapa & 1993 & -17.5 & -65.7 & 3430 & Cultivar adaptation & 2 & 3 & 4 & 7 & tu,le,st,ro, & $\mathrm{CL}$ & Condori et al. (2010) \\
\hline China, Huhhot & 1996 & 40.5 & 111.4 & 1065 & Partitioning & 1 & 1 & & 6 & tu,LAI & SL & Gao et al. (2003) \\
\hline China, Huhhot & 1998 & 40.5 & 111.4 & 1065 & Partitioning & 1 & 2 & & 6 & tu,le,st,to & SL & Liu et al. (2003b) \\
\hline China, Jining & 1999 & 41.0 & 113.0 & & Partitioning & 1 & 2 & & 6 & tu,le,st,to & SL & Gao et al. (2004) \\
\hline China, Zhalan & 1997 & 48.0 & 123.0 & & Various & 1 & 2 & & 7 & tu,le,st,to & SL & Liu et al. (2003a) \\
\hline China, Zhalan & 1998 & 48.0 & 123.0 & & Partitioning & 1 & 3 & & 7 & tu,le,st,to & $\mathrm{SL}$ & Gao et al. (2004) \\
\hline Colombia, Cundinamarca & 1999 & 4.4 & -74.1 & & Model validation & 1 & 1 & & 9 & tu,le,st,to,LAI & SiL & Forero Hernandez and Garzon Montaño (2000) \\
\hline Denmark, Jyndevad & $1982-1983$ & 54.9 & 9.1 & 10 & Various & 3 & 4 & & $14-15$ & tu,to & $S$ & Jørgensen (1984) \\
\hline Denmark, Jyndevad & 1990-1993 & 54.9 & 9.1 & 10 & $\mathrm{~N}$ rates & 4 & 1 & & $6-7$ & tu,ro,to,tuN,roN,toN & $\mathrm{S}$ & Edlefsen (1991) \\
\hline Denmark, Jyndevad & 1984-1986 & 54.9 & 9.1 & 10 & $\mathrm{~N}$ rates & 2 & 1 & & $5-7$ & tu,to,tuN & S & Jørgensen and Edlefsen (1987) \\
\hline Denmark, Tylstrup & $1981-1983$ & 57.2 & 10.0 & 10 & Various & 4 & 4 & & $13-14$ & tu,to & S & Bach and Nielsen (1985) \\
\hline Ecuador, San Gabriel & 1985 & 0.6 & -77.8 & & $\mathrm{~N}$ rates & 3 & 2 & & 4 & tu & SL & Clavijo Ponce (1999) \\
\hline Finland, Jokioinen & 1998-1999 & 60.8 & 23.5 & 84 & $\mathrm{CO}_{2} \mathrm{OTC}$ & 1 & 1 & 3 & 2 & tu,le,st & SL & De Temmerman et al. (2002a) \\
\hline Germany, Giessen & 1998-1999 & 50.6 & 8.7 & 68 & $\mathrm{CO}_{2} \mathrm{OTC}$ & 1 & 1 & $1-6$ & 2 & tu,le,st,to,LAI & SL & De Temmerman et al. (2002a) \\
\hline Germany, Giessen & 1998-1999 & 50.6 & 8.7 & 68 & $\mathrm{CO}_{2} \mathrm{FACE}$ & 1 & 1 & $2-6$ & 2 & tu,le,st,to,LAI & SL & De Temmerman et al. (2002a) \\
\hline India, Ludhiana & 2008-2011 & 30.9 & 75.8 & 244 & $\mathrm{~N}$ rates and irrigation & 4 & 1 & & 1 & tuf & $\mathrm{SL}$ & Arora et al. (2013) \\
\hline India, Modipuran & $2002-2009$ & 28.7 & 77.2 & 228 & Various & 1 & 1 & & 1 & tuf & L,SiCL & \\
\hline Ireland, Carlow & 1998-1999 & 52.9 & -6.9 & 57 & $\mathrm{CO}_{2} \mathrm{OTC}$ & 1 & 1 & 3 & 2 & tu,le,st,to & $\mathrm{SiC}$ & De Temmerman et al. (2002a) \\
\hline Italy, Rapolano & 1998-1999 & 42.7 & 11.9 & 38 & $\mathrm{CO}_{2} \mathrm{FACE}$ & 1 & 1 & $3-4$ & 2 & tu,le,st,to,LAl & SL & De Temmerman et al. (2002a) \\
\hline Peru, La Molina & 2003-2010 & -12.1 & -77.0 & 244 & Cultivar adaptation & 1 & 1 & & 1 & tuf & SL & \\
\hline Peru, La Molina & 2013 & -12.1 & -77.0 & 244 & Cultivar adaptation & 1 & 3 & 3 & 5 & tu,le,st & SL & \\
\hline
\end{tabular}




\begin{tabular}{|c|c|c|c|c|c|c|c|c|c|c|c|c|c|c|c|c|c|}
\hline Peru, La Molina & 1985 & -12.1 & -77.0 & 244 & \multicolumn{2}{|l|}{ Cultivar adaptation } & 2 & 3 & & 5 & \multicolumn{2}{|l|}{ tu,le,st,to,LAl } & \multicolumn{2}{|c|}{ SL, SCL,SL } & \multicolumn{3}{|c|}{ Trebejo and Midmore (1990) } \\
\hline Peru, San Ramon & 1984 & -11.1 & -75.3 & 800 & \multicolumn{2}{|c|}{ High temperature tolerance } & 1 & 3 & & 4 & \multicolumn{2}{|l|}{ tu,le,st,to } & \multicolumn{2}{|l|}{ SL } & \multicolumn{3}{|c|}{ Nelson (1987) } \\
\hline Peru, San Ramon & 2013 & -11.1 & -75.3 & 800 & \multicolumn{2}{|c|}{ High temperature tolerance } & 1 & 3 & 3 & 5 & \multicolumn{2}{|l|}{ tu,le,st,to } & \multicolumn{2}{|l|}{ SL } & & & \\
\hline Scotland, Dundee & 1984-1985 & 56.5 & -3.1 & 40 & \multicolumn{2}{|l|}{$\mathrm{N}$ rates } & 2 & 1 & & 8 & tu,le,st,to,LAI,de,tur & leN,stN,toN & SL & & Marshall a & Van Den Bro & (1995) \\
\hline Scotland, Dundee & $1986-1987$ & 56.5 & -3.1 & 40 & Irrigation & & 4 & 1 & & 8 & tu,le,st,to,LAI,SWC & & SL & & Marshall a & Van Den Bro & (1995) \\
\hline Sweden, Goteborg & 1998-1999 & 57.9 & 12.4 & 58 & $\mathrm{CO}_{2}$ OTC & & 1 & 1 & $4-6$ & 2 & tu,le,st,to & & SL & & De Temme & man et al. (200 & \\
\hline Uganda, Kalengyere & $2001-2009$ & -1.2 & 29.8 & $2400 \mathrm{~V}$ & Various & & 1 & 1 & & 1 & tuf & & C & & & & \\
\hline United Kingdom, Sutton & 1998-1999 & 52.8 & -1.3 & 87 & $\mathrm{CO}_{2} \mathrm{OTC}$ & & 1 & 1 & 3 & 2 & tu,le,st,to,LAl & & SL & & & & \\
\hline United States, Benton & 2003 & 45.9 & -119.5 & & $\mathrm{~N}$ rates & & 2 & 1 & & $5-6$ & tu,le,st,to,LAI,tuN,le & ,stN,toN & $\mathrm{S}$ & & Alva et al. & 010) & \\
\hline United States, Hastings & $2011-2012$ & 29.7 & -81.5 & 2 & $\mathrm{~N}$ rates & & 2 & 3 & 3 & 1 & tu,le,st,tuN,leN,stN, & $\mathrm{N}, \mathrm{soN}$ & $S$ & & Zotarelli e & I. (2014) & \\
\hline United States, New York & 1980 & 42.4 & -76.5 & & Various & & 1 & 1 & & 5 & tu,le,st,to & & SL & & Hoogenbo & $n$ et al. (2012) & \\
\hline United States, Idaho & 1988 & 45.8 & -119.3 & & Various & & 1 & & & $9-15$ & tu,le,st,to,LAI & & SL & & Hoogenbo & $\mathrm{n}$ et al. (2012) & \\
\hline United States, Suwanee & 2010-2013 & 30.1 & -83.1 & $13.7 \mathrm{~N}$ & $\mathrm{~N}$ mass balance & & 1 & 1 & 12 & 1 & tuf & & $\mathrm{s}$ & & Prasad et & (2015) & \\
\hline United States, Suwanee & 2001 & 30.1 & -83.1 & $13.7 \mathrm{~N}$ & $\mathrm{~N}$ mass balance & & 1 & 1 & 4 & $3-5$ & tu,le,st,soN,SWC & & $S$ & & Albert $(20$ & & \\
\hline United States, Suwanee & 2003 & 30.1 & -83.1 & $13.7 \mathrm{~N}$ & $\mathrm{~N}$ mass balance & & 1 & 1 & 8 & $3-5$ & tu,le,st,soN,SWC & & $\mathrm{S}$ & & Warren (2 & & \\
\hline $\begin{array}{c}(\mathrm{B}) \\
\text { Location }\end{array}$ & Year & $N \operatorname{tr}^{*}$ & & $\begin{array}{l}\text { owing date } \\
\text { DOY }\end{array}$ & $\begin{array}{l}\text { Emergence } \\
\text { date } \\
\text { DOY }\end{array}$ & Harv & $\begin{array}{l}\text { est d } \\
\text { OOY }\end{array}$ & & $\mathrm{N}$ ap & oplication $\left(\mathrm{kg}^{-} \mathrm{ha}^{-1}\right)^{\star *}$ & Irrigation (mm) & Rainfall ( & & Type & f irrigation & $\mathrm{CO}_{2}(\mathrm{ppm})$ & Cultivar \\
\hline Argentina, Balcarce & 1991 & 1 & 298 & & n.a. & 64 & & & 0 & & 113 & 540 & & Mixed & & Default & Spunta \\
\hline & & 2 & 298 & & n.a. & 64 & & & 60_(1) & & 113 & 540 & & Mixed & & Default & Spunta \\
\hline & & 3 & 298 & & n.a. & 64 & & & $120(1)$ & & 113 & 540 & & Mixed & & Default & Spunta \\
\hline & & 4 & 298 & & n.a. & 64 & & & 160 (1) & & 113 & 540 & & Mixed & & Default & Spunta \\
\hline Australia & 1970 & 1 & 222 & & 273 & 356 & & & 425_(2) & & n.a. & 219 & & Full & & Default & Sebago \\
\hline & & 2 & 222 & & 273 & 356 & & & 425_(2) & & n.a. & 219 & & Full & & Default & Sebago \\
\hline & & 3 & 222 & & 273 & 356 & & & $425(2)$ & & n.a. & 219 & & Full & & Default & Sebago \\
\hline Belgium, Tervuren & $1998+$ & $1^{\mathrm{N}}$ & 127 & & 135 & 257 & & & 205_(2) & & 373 & 423 & & Mixed & & 380 & Bintje \\
\hline & & $2^{N}$ & 127 & & 135 & 257 & & & $205(2)$ & & 373 & 423 & & Mixed & & 386 & Bintje \\
\hline & & $3^{N}$ & 127 & & 135 & 257 & & & 205_(2) & & 373 & 423 & & Mixed & & 676 & Bintje \\
\hline & $1999+$ & $1^{\mathrm{N}}$ & 131 & & 144 & 250 & & & 220 (2) & & 182 & 204 & & Full & & 365 & Bintje \\
\hline & & $2^{N}$ & 131 & & 144 & 250 & & & 220 (2) & & 181 & 204 & & Full & & 370 & Bintje \\
\hline & & $3^{N}$ & 131 & & 144 & 250 & & & 220 (2) & & 183 & 204 & & Mixed & & 664 & Bintje \\
\hline Bolivia, Belen & 1997 & 1 & 288 & & 316 & 84 & & & $110(1)$ & & n.a. & 264 & & Full & & Default & Waycha \\
\hline & & 2 & 288 & & 316 & 84 & & & 110 (1) & & n.a. & 264 & & Full & & Default & Lucky \\
\hline Bolivia, Chinoli & 1997 & 1 & 301 & & 340 & 62 & & & 124_(1) & & n.a. & 275 & & Full & & Default & Desiree \\
\hline
\end{tabular}




\begin{tabular}{|c|c|c|c|c|c|c|c|c|c|c|c|}
\hline \multirow[t]{3}{*}{ Bolivia, Koari } & 1997 & 1 & 281 & 329 & $\mid 111$ & $100(1)$ & - & 540 & Rainfed & Default & Waychacal \\
\hline & & 2 & 281 & 329 & 111 & $100(1)$ & - & 540 & Rainfed & Default & Alphacal \\
\hline & & 3 & 281 & 329 & 111 & $100(1)$ & - & 540 & Rainfed & Default & Luckycal $^{\text {cal }}$ \\
\hline \multirow[t]{2}{*}{ Bolivia, Patacamaya } & 1997 & $1^{\mathrm{N}}$ & 300 & 351 & 112 & $110(1)$ & n.a. & 341 & Full & Default & Waycha \\
\hline & & $2^{N}$ & 300 & 351 & 112 & $110(1)$ & n.a. & 341 & Full & Default & Lucky \\
\hline \multirow[t]{2}{*}{ Bolivia, Patacamaya } & $1998-1$ & 1 & 292 & 347 & 110 & 110 & n.a. & 334 & Full & Default & Waycha \\
\hline & & 2 & 292 & 347 & 110 & $110(1)$ & n.a. & 334 & Full & Default & Lucky \\
\hline \multirow[t]{2}{*}{ Bolivia, Patacamaya } & $1998-2$ & $1^{\mathrm{N}}$ & 292 & 342 & 110 & $110(1)$ & n.a. & 334 & Full & Default & Waycha \\
\hline & & $2^{N}$ & 292 & 342 & 110 & $110(1)$ & n.a. & 334 & Full & Default & Lucky \\
\hline \multirow[t]{6}{*}{ Bolivia, Toralapa } & 1993 & $1^{\mathrm{N}}$ & 295 & 351 & 103 & $120(1)$ & n.a. & 440 & Full & Default & Waycha \\
\hline & & $2^{N}$ & 295 & 351 & 103 & $120(1)$ & n.a. & 440 & Full & Default & Alpha \\
\hline & & $3^{N}$ & 295 & 351 & 103 & $120(1)$ & n.a. & 440 & Full & Default & Lucky \\
\hline & & $4^{N}$ & 295 & 351 & 103 & $120(1)$ & - & 440 & Rainfed & Default & Waycha \\
\hline & & $5^{N}$ & 295 & 351 & 103 & $120(1)$ & - & 440 & Rainfed & Default & Alpha \\
\hline & & $6^{N}$ & 295 & 351 & 103 & $120(1)$ & - & 440 & Rainfed & Default & Lucky \\
\hline China, Huhhot & 1996 & 1 & 118 & n.a. & 250 & $150(2)$ & n.a. & 252 & Full & Default & Desiree \\
\hline China, Huhhot & 1998 & 1 & 115 & n.a. & 253 & $150(2)$ & n.a. & 470.9 & Full & Default & Desiree \\
\hline \multirow[t]{3}{*}{ China, Jining } & 1999 & 1 & 119 & n.a. & 234 & 74 & n.a. & 156 & Full & Default & Desiree \\
\hline & & 2 & 119 & n.a. & 234 & 74 & n.a. & 156 & Full & Default & Kexin $1^{\text {cal }}$ \\
\hline & & 3 & 119 & n.a. & 234 & $74 \_(2)$ & n.a. & 156 & Full & Default & Jinguancal \\
\hline China, Zhalan & 1997 & 1 & 119 & n.a. & 253 & 180 & n.a. & 89 & Full & Default & Desiree \\
\hline \multirow[t]{2}{*}{ China, Zhalan } & 1998 & 1 & 119 & n.a. & 242 & $90 \_(2)$ & - & 754 & Rainfed & Default & Kexin 1 \\
\hline & & 2 & 119 & n.a. & 242 & $90(2)$ & - & 754 & Rainfed & Default & Neishu $7^{\text {cal }}$ \\
\hline Colombia, Cundinamarca & 1999 & 1 & 119 & 143 & 262 & $100(1)$ & - & 392 & Rainfed & Default & Capiro \\
\hline \multirow[t]{3}{*}{ Denmark, Jyndevad } & 1981 & 1 & 119 & 149 & 225 & $155(1)$ & 169 & 431 & Mixed & Default & Bintje \\
\hline & 1982 & 1 & 119 & 148 & 236 & $155(1)$ & 222 & 521 & Mixed & Default & Bintje \\
\hline & 1983 & 1 & 122 & 148 & 215 & 155 & 156 & 370 & Mixed & Default & Bintje \\
\hline \multirow[t]{5}{*}{ Denmark, Jyndevad } & 1990 & 1 & 107 & n.a. & 267 & $180(1)$ & 107 & 499 & Mixed & Default & Bintje $^{\text {cal }}$ \\
\hline & & 2 & 107 & n.a. & 267 & $180(4)$ & 104 & 499 & Mixed & Default & Bintje $\mathrm{cal}^{\text {cal }}$ \\
\hline & & 3 & 107 & n.a. & 267 & $180(4)$ & 105 & 499 & Mixed & Default & Bintje cal \\
\hline & 1991 & 1 & 101 & 149 & 273 & $180(1)$ & 137 & 390 & Mixed & Default & Bintje \\
\hline & & 2 & 101 & 149 & 273 & $180(3)$ & 137 & 390 & Mixed & Default & Bintje \\
\hline
\end{tabular}




\begin{tabular}{|c|c|c|c|c|c|c|c|c|c|c|c|}
\hline & & 3 & 101 & 149 & 273 & $180(3)$ & 137 & 390 & Mixed & Default & Bintje \\
\hline & 1992 & 1 & 100 & 139 & 224 & $180(1)$ & 231 & 195 & Mixed & Default & Bintje \\
\hline & & 2 & 100 & 139 & 224 & $180(3)$ & 231 & 195 & Mixed & Default & Bintje \\
\hline & & 3 & 100 & 139 & 224 & $180(4)$ & 231 & 195 & Mixed & Default & Bintje \\
\hline & 1993 & 1 & 110 & 133 & 263 & $180(1)$ & 70 & 350 & Mixed & Default & Bintje \\
\hline & & 2 & 110 & 133 & 263 & $180(3)$ & 70 & 350 & Mixed & Default & Bintje \\
\hline & & 3 & 110 & 133 & 263 & $180(4)$ & 70 & 350 & Mixed & Default & Bintje \\
\hline \multirow[t]{6}{*}{ Denmark, Jyndevad } & 1984 & 1 & 117 & 151 & 278 & $150(1)$ & 71 & 519 & Mixed & Default & Tilva ${ }^{\text {cal }}$ \\
\hline & & 2 & 117 & 151 & 278 & $200(1)$ & 71 & 519 & Mixed & Default & Tilva \\
\hline & 1985 & 1 & 119 & 149 & 270 & $150(1)$ & 27 & 465 & Mixed & Default & Tilva \\
\hline & & 2 & 119 & 149 & 270 & $200(1)$ & 27 & 465 & Mixed & Default & Tilva \\
\hline & 1986 & 1 & 118 & 146 & 293 & $150(1)$ & 186 & 488 & Mixed & Default & Tilva \\
\hline & & 2 & 118 & 146 & 293 & $200(1)$ & 186 & 488 & Mixed & Default & Tilva \\
\hline \multirow[t]{15}{*}{ Denmark, Tylstrup } & 1981 & 1 & 120 & 155 & 272 & $140(1)$ & 25 & 405 & Mixed & Default & Bintje \\
\hline & & 2 & 120 & 152 & 272 & $140(1)$ & 25 & 405 & Mixed & Default & Sava ${ }^{\text {cal }}$ \\
\hline & & 3 & 120 & 149 & 272 & $140(1)$ & 25 & 405 & Mixed & Default & Posmo cal \\
\hline & & 4 & 120 & 150 & 272 & $140(1)$ & 25 & 405 & Mixed & Default & Kaptah ${ }^{\text {cal }}$ \\
\hline & & 5 & 120 & 145 & 272 & $140(1)$ & 25 & 405 & Mixed & Default & Dianella ${ }^{\text {cal }}$ \\
\hline & 1982 & 1 & 109 & 149 & 270 & $180(1)$ & 185 & 607 & Mixed & Default & Bintje \\
\hline & & 2 & 109 & 155 & 270 & $180(1)$ & 185 & 607 & Mixed & Default & Sava \\
\hline & & 3 & 109 & 152 & 270 & $180(1)$ & 185 & 607 & Mixed & Default & Posmo \\
\hline & & 4 & 109 & 152 & 270 & $180(1)$ & 185 & 607 & Mixed & Default & Kaptah \\
\hline & & 5 & 109 & 149 & 298 & $180(1)$ & 185 & 607 & Mixed & Default & Dianella \\
\hline & 1983 & 1 & 109 & 154 & 298 & $160(2)$ & 135 & 566 & Mixed & Default & Bintje \\
\hline & & 2 & 109 & 154 & 298 & $160(2)$ & 135 & 566 & Mixed & Default & Sava \\
\hline & & 3 & 109 & 150 & 298 & $160(2)$ & 135 & 566 & Mixed & Default & Posmo \\
\hline & & 4 & 109 & 154 & 298 & $160(2)$ & 135 & 566 & Mixed & Default & Kaptah \\
\hline & & 5 & 109 & 149 & 298 & $160(2)$ & 135 & 566 & Mixed & Default & Dianella \\
\hline \multirow[t]{4}{*}{ Ecuador, San Gabriel } & 1985 & 1 & 45 & 76 & 241 & 168_(2) & - & 426 & Rainfed & Default & INIAP-maria \\
\hline & & 2 & 45 & 76 & 241 & 117_(1) & - & 426 & Rainfed & Default & INIAP-gabriela \\
\hline & & 3 & 45 & 76 & 241 & $168(2)$ & - & 426 & Rainfed & Default & INIAP-maria \\
\hline & & 4 & 45 & 76 & 241 & 117_(1) & - & 426 & Rainfed & Default & INIAP-gabriela \\
\hline Finland, Jokioinen & $1998+$ & 1 & 152 & 163 & 270 & 80_(2) & - & 329 & Full & 375 & Bintje \\
\hline
\end{tabular}




\begin{tabular}{|c|c|c|c|c|c|c|c|c|c|c|c|}
\hline & $1999+$ & 1 & 149 & 159 & 264 & $80 \_(2)$ & - & 122 & Full & 550 & Bintje \\
\hline \multirow[t]{6}{*}{ Germany, Giessen } & $1998+$ & $1^{\mathrm{N}}$ & 124 & 134 & 250 & $150(2)$ & 67 & 365 & Mixed & 373 & Bintje \\
\hline & & $2^{N}$ & 124 & 134 & 250 & $150(2)$ & 67 & 365 & Mixed & 541 & Bintje \\
\hline & & $3^{N}$ & 124 & 134 & 250 & $150(2)$ & 67 & 365 & Mixed & 690 & Bintje \\
\hline & $1999+$ & $1^{\mathrm{N}}$ & 130 & 147 & 258 & $116(2)$ & 152 & 267 & Full & 380 & Bintje \\
\hline & & $2^{N}$ & 130 & 147 & 258 & $116(2)$ & 152 & 267 & Full & 541 & Bintje \\
\hline & & $3^{N}$ & 130 & 147 & 258 & $116(2)$ & 148 & 267 & Full & 708 & Bintje \\
\hline \multirow[t]{4}{*}{ Germany, Giessen } & $1998++$ & $1^{N}$ & 125 & 135 & 257 & $150(2)$ & 186 & 417 & Mixed & 401 & Bintje \\
\hline & & $2^{N}$ & 125 & 135 & 257 & $150(2)$ & 186 & 417 & Mixed & 429 & Bintje \\
\hline & $1999++$ & 1 & 130 & 145 & 239 & $156 .(2)$ & 128 & 250 & Full & 374 & Bintje \\
\hline & & 2 & 130 & 145 & 239 & $156(2)$ & 126 & 250 & Full & 491 & Bintje \\
\hline \multirow[t]{22}{*}{ India, Ludhiana } & 2008 & 1 & 290 & n.a. & 29 & 0 & 80 & 18 & Mixed & Default & Kufri Bahar \\
\hline & & 2 & 290 & n.a. & 29 & $136(2)$ & 80 & 18 & Mixed & Default & Kufri Bahar \\
\hline & & 3 & 290 & n.a. & 29 & $180(2)$ & 80 & 18 & Mixed & Default & Kufri Bahar \\
\hline & & 4 & 290 & n.a. & 29 & 224_(2) & 80 & 18 & Mixed & Default & Kufri Bahar \\
\hline & & 5 & 290 & n.a. & 29 & 0 & 160 & 18 & Mixed & Default & Kufri Bahar \\
\hline & & 6 & 290 & n.a. & 29 & $136(2)$ & 160 & 18 & Mixed & Default & Kufri Bahar \\
\hline & & 7 & 290 & n.a. & 29 & $180(2)$ & 160 & 18 & Mixed & Default & Kufri Bahar \\
\hline & & 8 & 290 & n.a. & 29 & 224_(2) & 160 & 18 & Mixed & Default & Kufri Bahar \\
\hline & & 9 & 290 & n.a. & 29 & 0 & 200 & 18 & Mixed & Default & Kufri Bahar \\
\hline & & 10 & 290 & n.a. & 29 & $136(2)$ & 200 & 18 & Mixed & Default & Kufri Bahar \\
\hline & & 11 & 290 & n.a. & 29 & $180(2)$ & 200 & 18 & Mixed & Default & Kufri Bahar \\
\hline & & 12 & 290 & n.a. & 29 & 224_(2) & 200 & 18 & Mixed & Default & Kufri Bahar \\
\hline & 2010 & 1 & 285 & n.a. & 29 & 0 & 80 & 32 & Mixed & Default & Kufri Bahar \\
\hline & & 2 & 285 & n.a. & 29 & $136(2)$ & 80 & 32 & Mixed & Default & Kufri Bahar \\
\hline & & 3 & 285 & n.a. & 29 & $180(2)$ & 80 & 32 & Mixed & Default & Kufri Bahar \\
\hline & & 4 & 285 & n.a. & 29 & 224_(2) & 80 & 32 & Mixed & Default & Kufri Bahar \\
\hline & & 5 & 285 & n.a. & 29 & 0 & 160 & 32 & Mixed & Default & Kufri Bahar \\
\hline & & 6 & 285 & n.a. & 29 & $136(2)$ & 160 & 32 & Mixed & Default & Kufri Bahar \\
\hline & & 7 & 285 & n.a. & 29 & $180(2)$ & 160 & 32 & Mixed & Default & Kufri Bahar \\
\hline & & 8 & 285 & n.a. & 29 & 224_(2) & 160 & 32 & Mixed & Default & Kufri Bahar \\
\hline & & 9 & 285 & n.a. & 29 & 0 & 200 & 32 & Mixed & Default & Kufri Bahar \\
\hline & & 10 & 285 & n.a. & 29 & 136_(2) & 200 & 32 & Mixed & Default & Kufri Bahar \\
\hline
\end{tabular}




\begin{tabular}{|c|c|c|c|c|c|c|c|c|c|c|c|}
\hline & & 11 & 285 & n.a. & 29 & $180(2)$ & 200 & 32 & Mixed & Default & Kufri Bahar \\
\hline & & 12 & 285 & n.a. & 29 & 224_(2) & 200 & 32 & Mixed & Default & Kufri Bahar \\
\hline \multirow[t]{8}{*}{ India, Modipuran } & 2002 & 1 & 288 & n.a. & 3 & 181 (2) & n.a. & 0 & Full & Default & Kufri Bahar \\
\hline & 2003 & 1 & 299 & n.a. & 18 & 181_(2) & n.a. & 30 & Full & Default & Kufri Bahar \\
\hline & 2004 & 1 & 293 & n.a. & 11 & 181_(2) & n.a. & 0 & Full & Default & Kufri Bahar \\
\hline & 2005 & 1 & 289 & n.a. & 8 & 181_(2) & n.a. & 1 & Full & Default & Kufri Bahar \\
\hline & 2006 & 1 & 294 & n.a. & 13 & 181_(2) & n.a. & 0 & Full & Default & Kufri Bahar \\
\hline & 2007 & 1 & 298 & n.a. & 17 & 181_(2) & n.a. & 0 & Full & Default & Kufri Bahar \\
\hline & 2008 & 1 & 290 & n.a. & 8 & 181_(2) & n.a. & 12 & Full & Default & Kufri Bahar \\
\hline & 2009 & 1 & 302 & n.a. & 21 & 181 (2) & n.a. & 0 & Full & Default & Kufri Bahar \\
\hline \multirow[t]{4}{*}{ Ireland, Carlow } & $1998^{N}$ & 1 & 128 & 138 & 280 & 250 & 61 & 343 & Full & 372 & Bintje \\
\hline & & 2 & 128 & 138 & 280 & 250 & 31 & 343 & Full & 693 & Bintje \\
\hline & $1999^{N}$ & 1 & 140 & 152 & 249 & 250 & 89 & 392 & Full & 372 & Bintje \\
\hline & & 2 & 140 & 152 & 249 & 250 & 91 & 392 & Full & 670 & Bintje \\
\hline \multirow[t]{6}{*}{ Italy, Rapolano } & $1998++$ & 1 & 141 & 149 & 237 & 240 & 309 & 554 & Full & 366 & Bintje \\
\hline & & 2 & 141 & 149 & 237 & 240 & 294 & 554 & Full & 552 & Bintje \\
\hline & & 3 & 141 & 149 & 237 & 240 & 285 & 554 & Full & 367 & Bintje \\
\hline & $1999++$ & 1 & 126 & 147 & 237 & 250 & 462 & 146 & Mixed & 367 & Bintie \\
\hline & & 2 & 126 & 147 & 237 & 250 & 462 & 146 & Mixed & 552 & Bintie \\
\hline & & 3 & 126 & 147 & 237 & 250 & 462 & 146 & Mixed & 367 & Bintie \\
\hline \multirow[t]{8}{*}{ Peru, La Molina } & 2003 & 1 & 181 & n.a. & 314 & 310 & 450 & 0 & Irrigated & Default & Amarilis \\
\hline & 2004 & 1 & 177 & n.a. & 307 & $310 .(2)$ & 350 & 0 & Irrigated & Default & Amarilis \\
\hline & 2005 & 1 & 164 & n.a. & 281 & 298_(2) & 450 & 0 & Irrigated & Default & Amarilis \\
\hline & 2006 & 1 & 186 & n.a. & 319 & $310 .(2)$ & 350 & 0 & Irrigated & Default & Amarilis \\
\hline & 2007 & 1 & 152 & n.a. & 275 & $350 .(2)$ & 400 & 0 & Irrigated & Default & Amarilis \\
\hline & 2008 & 1 & 182 & n.a. & 294 & 235_(2) & 350 & 0 & Irrigated & Default & Amarilis \\
\hline & 2009 & 1 & 183 & n.a. & 292 & 235_(2) & 400 & 0 & Irrigated & Default & Amarilis \\
\hline & 2010 & 1 & 196 & n.a. & 307 & 227_(2) & 350 & 0 & Irrigated & Default & Amarilis \\
\hline \multirow[t]{3}{*}{ Peru, La Molina } & 2013 & 1 & 179 & 200 & 288 & 210 & 215 & 14 & Full & Default & Achirana \\
\hline & & 2 & 179 & 198 & 288 & 210 & 215 & 14 & Full & Default & Atlantic \\
\hline & & 3 & 179 & 198 & 288 & 210 & 215 & 14 & Full & Default & Sarnaveal \\
\hline \multirow[t]{2}{*}{ Peru, La Molina } & 1985 & 1 & 30 & 60 & 122 & 160 & 449 & 3 & Irrigated & Default & DTO-33 \\
\hline & & 2 & 30 & 60 & 130 & 160 & 480 & 3 & Irrigated & Default & LT1 \\
\hline
\end{tabular}




\begin{tabular}{|c|c|c|c|c|c|c|c|c|c|c|c|}
\hline & & 3 & 30 & 60 & 144 & $160(2)$ & 485 & 3 & Irrigated & Default & Revolucion \\
\hline & & 4 & 176 & 206 & 285 & $160 \_(2)$ & 315 & 1 & Irrigated & Default & DTO-33 \\
\hline & & 5 & 176 & 206 & 291 & $160(2)$ & 257 & 1 & Irrigated & Default & LT1 \\
\hline & & 6 & 176 & 206 & 274 & $160 \_(2)$ & 272 & 1 & Irrigated & Default & Revolucion \\
\hline \multirow[t]{3}{*}{ Peru, San Ramon } & 1984 & 1 & 109 & 122 & 199 & $200(2)$ & n.a. & 336 & Full & Default & DTO-33 \\
\hline & & 2 & 109 & 126 & 199 & $200(2)$ & n.a. & 336 & Full & Default & Desiree \\
\hline & & 3 & 109 & 128 & 199 & 200 (2) & n.a. & 336 & Full & Default & Revolucion \\
\hline \multirow[t]{3}{*}{ Peru, San Ramon } & 2013 & 1 & 213 & 228 & 301 & 300 (2) & 449 & 303 & Mixed & Default & Achirana ${ }^{\text {cal }}$ \\
\hline & & 2 & 213 & 228 & 301 & $300(2)$ & 449 & 303 & Mixed & Default & Atlantic \\
\hline & & 3 & 213 & 228 & 301 & $300 \_(2)$ & 449 & 303 & Mixed & Default & Sarnav \\
\hline \multirow[t]{6}{*}{ Scotland, Dundee } & 1984 & $1^{\mathrm{N}}$ & 104 & 150 & 268 & 0 & 187 & 202 & Mixed & Default & Maris piper \\
\hline & & $2^{N}$ & 104 & 150 & 268 & 240 & 187 & 202 & Mixed & Default & Maris piper \\
\hline & 1985 & $1^{N}$ & 114 & 154 & 262 & 0 & 15 & 373 & Mixed & Default & Maris piper \\
\hline & & $2^{N}$ & 114 & 154 & 262 & 240_(1) & 15 & 373 & Mixed & Default & Maris piper \\
\hline & 1986 & $1^{N}$ & 135 & 166 & 266 & 175_(1) & 91 & 200 & Mixed & Default & Maris piper \\
\hline & 1987 & $1^{\mathrm{N}}$ & 119 & 155 & 258 & $175_{-}(1)$ & 39 & 346 & Mixed & Default & Maris piper \\
\hline \multirow[t]{2}{*}{ Sweden, Goteborg } & $1998+$ & $1^{\mathrm{N}}$ & 145 & 154 & 252 & 88_(2) & 480 & 357 & Mixed & 708 & Bintje \\
\hline & & $2^{N}$ & 145 & 155 & 252 & 88_(2) & 480 & 357 & Mixed & 404 & Bintje \\
\hline \multirow[t]{4}{*}{ Uganda, Kalengyere } & 2004 & 1 & 61 & n.a. & 169 & $100 \_(1)$ & - & 294 & Rainfed & Default & Asante \\
\hline & 2005 & 1 & 80 & n.a. & 189 & $100(1)$ & - & 318 & Rainfed & Default & Asante \\
\hline & 2006 & 1 & 82 & n.a. & 186 & $100 \_(1)$ & - & 399 & Rainfed & Default & Asante \\
\hline & 2009 & 1 & 273 & n.a. & 355 & 120 & - & 501 & Rainfed & Default & Asante \\
\hline \multirow[t]{6}{*}{ United Kingdom, Sutton } & $1998+$ & $1^{\mathrm{N}}$ & 126 & 136 & 239 & $110(2)$ & 98 & 251 & Full & 379 & Bintje \\
\hline & & $2^{N}$ & 126 & 136 & 239 & $110 \_(2)$ & 97 & 251 & Full & 563 & Bintje \\
\hline & & $3^{N}$ & 126 & 136 & 239 & $110(2)$ & 99 & 251 & Full & 673 & Bintje \\
\hline & $1999+$ & $1^{N}$ & 132 & 144 & 249 & $250 \_(2)$ & 131 & 247 & Full & 399 & Bintje \\
\hline & & $2^{N}$ & 132 & 144 & 249 & $250 \_(2)$ & 134 & 247 & Full & 543 & Bintje \\
\hline & & $3^{N}$ & 132 & 144 & 249 & $250 \_(2)$ & 132 & 247 & Full & 694 & Bintje \\
\hline \multirow[t]{2}{*}{ United States, Benton } & 2003 & 1 & 87 & 110 & 231 & 324_(9) & 666 & 164 & Mixed & Default & Russet Burbank \\
\hline & & 2 & 87 & 110 & 231 & $669(5)$ & 666 & 164 & Mixed & Default & Russet Burbank \\
\hline \multirow[t]{3}{*}{ United States, Hastings } & 2011 & 1 & 12 & 43 & 108 & 168_(3) & n.a. & 255 & Full & Default & Atlantic \\
\hline & & 2 & 12 & 43 & 108 & 224_(3) & n.a. & 258 & Full & Default & Atlantic \\
\hline & & 3 & 19 & 45 & 117 & 168_(3) & n.a. & 258 & Full & Default & Atlantic \\
\hline
\end{tabular}




\begin{tabular}{|c|c|c|c|c|c|c|c|c|c|c|c|}
\hline & & 4 & 19 & 45 & 117 & 224_(3) & n.a. & 258 & Full & Default & Atlantic \\
\hline & & 5 & 20 & 45 & 116 & 168(3) & n.a. & 258 & Full & Default & Atlantic \\
\hline & & 6 & 20 & 45 & 116 & 224_(3) & n.a. & 258 & Full & Default & Atlantic \\
\hline United States, New York & 1980 & 1 & 143 & 157 & 257 & 275 & 209 & 297 & Full & Default & Kathadin \\
\hline \multirow[t]{10}{*}{ United States, Idaho } & 1988 & 1 & 105 & 129 & 264 & 367_(10) & 669 & 136 & Full & Default & Russet Burbank \\
\hline & & 2 & 89 & 115 & 174 & 354 & 580 & 123 & Full & Default & Russet Burbank \\
\hline & & 3 & 98 & 118 & 174 & 433_(16) & 687 & 136 & Full & Default & Russet Burbank \\
\hline & & 4 & 117 & 134 & 264 & 349 & 644 & 136 & Full & Default & Russet Burbank \\
\hline & & 5 & 75 & 105 & 221 & 771 & 598 & 123 & Full & Default & Russet Burbank \\
\hline & & 6 & 81 & 114 & 250 & 375 & 669 & 123 & Full & Default & Russet Burbank \\
\hline & & 7 & 107 & 131 & 174 & 375 & 605 & 123 & Full & Default & Russet Burbank \\
\hline & & 8 & 91 & 120 & 174 & $175(7)$ & 686 & 135 & Full & Default & Russet Burbank \\
\hline & & 9 & 116 & 136 & 264 & 375 & 686 & 136 & Full & Default & Russet Burbank \\
\hline & & 10 & 98 & 125 & 174 & 375 & 740 & 136 & Full & Default & Russet Burbank \\
\hline \multirow[t]{6}{*}{ United States, Suwanee } & 2010 & 1 & 41 & 74 & 140 & 265 & 281 & 758 & Mixed & Default & Red Lasoda \\
\hline & 2011 & 1 & 28 & 57 & 118 & $278(5)$ & 297 & 537 & Mixed & Default & Red Lasoda \\
\hline & & 2 & 43 & 62 & 140 & 285 & 291 & 558 & Mixed & Default & Red Lasoda \\
\hline & 2012 & 1 & 31 & 51 & 123 & 285 & 349 & 320 & Mixed & Default & Red Lasoda \\
\hline & & 2 & 50 & 69 & 141 & $248(4)$ & 343 & 356 & Mixed & Default & Red Lasoda \\
\hline & 2013 & 1 & 45 & 65 & 140 & 248 & 287 & 448 & Mixed & Default & Red Lasoda \\
\hline \multirow[t]{5}{*}{ United States, Suwanee } & 2001 & 1 & 46 & 62 & 141 & $313(5)$ & 507 & 290 & Mixed & Default & Red Lasoda \\
\hline & & 2 & 46 & 62 & 141 & $280(5)$ & 507 & 290 & Mixed & Default & Red Lasoda \\
\hline & 2002 & 1 & 43 & 60 & 137 & $292(5)$ & 343 & 481 & Mixed & Default & Red Lasoda \\
\hline & & 2 & 46 & 64 & 138 & $261(5)$ & 272 & 489 & Mixed & Default & Red Lasoda \\
\hline & 2003 & 1 & 41 & 64 & 138 & 278_(4) & 242 & 576 & Mixed & Default & Red Lasoda \\
\hline
\end{tabular}

cal: Treatments used for calibration.

$\mathrm{N}$ : Treatments with estimated initial soil $\mathrm{N}$.

*: Number or treatments.

**: Number of nitrogen applications are in parenthesis.

+: OTC.

++: FACE.

n.a.: Not available.

-: Not applicable.

Full: Automatic irrigation. 
a Latitude.

${ }^{\mathrm{b}}$ Longitude.

${ }^{c}$ Altitude.

${ }^{d}$ FACE: Free-Air- $\mathrm{CO}_{2}$-Enrichment, OTC: Open Top Chambers.

${ }^{e} \mathrm{AN}$ umber of treatments.

${ }^{f} \mathrm{~A}$ Number of cultivars.

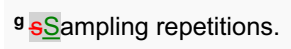

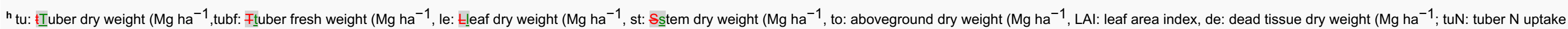
( $\mathrm{kg} \mathrm{ha}^{-1}$; le: Leaf $\mathrm{N}$ uptake $\left(\mathrm{kg} \mathrm{ha}^{-1}\right.$; stN: Stem $\mathrm{N}$ uptake ( $\mathrm{kg} \mathrm{ha}^{-1}$, toN: aboveground nitrogen uptake $\left(\mathrm{kg} \mathrm{ha}^{-1}\right)$, SoN: Soil $\mathrm{N}$ content (ppm), SWC: Soil water content ( $\mathrm{m}^{3} \mathrm{~m}^{-3}$.

'C: clay; S:sand; Si: silt; L:Ioam.

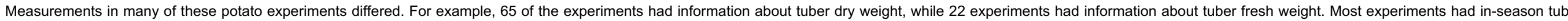

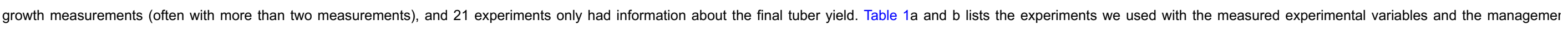
information.

\subsection{Weather data}

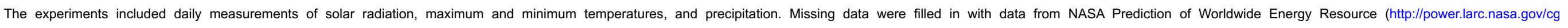
bin/cgiwrap/solar/agro.cgi).

\subsection{Soil parameters}

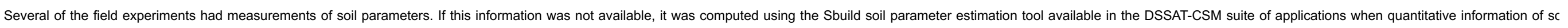
texture and organic carbon were available. Generic soil profiles, available in DSSAT-CSM, were assigned only when qualitative soil type information was available.

\subsection{Calibration of cultivar parameters}

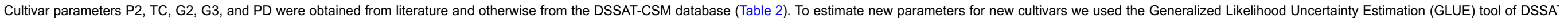

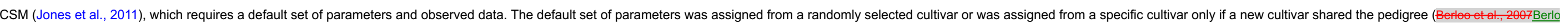

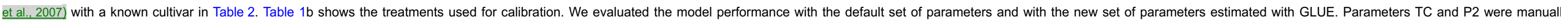

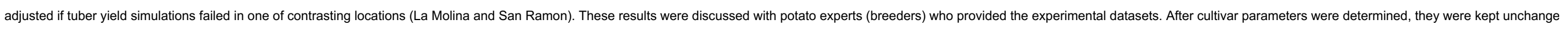
across experiments and locations. Table 2 lists the cultivar parameters used in simulations performed with the SUBSTOR-potato model.

Table 2 Cultivar parameters used in the SUBSTOR-potato model.

\begin{tabular}{|c|c|c|c|c|c|c|c|c|c|}
\hline Cultivar & Species & Maturity type & Country & $\mathrm{G} 2\left(\mathrm{~cm}^{2} \mathrm{~m}^{-2}\right.$ day $\left.^{-1}\right)$ & $\begin{array}{c}\mathrm{G} 3 \\
\left(\mathrm{~g} \mathrm{~m}^{-2} \mathrm{day}^{-1}\right)\end{array}$ & PD (-) & $\mathrm{P} 2(-)$ & $\operatorname{TC}\left({ }^{\circ} \mathrm{C}\right)$ & Source \\
\hline Achirana & S. tuberosum & Early & Peru & 2000 & 21 & 0.8 & 0.5 & 17 & \\
\hline Alpha & S. tuberosum & Late & Bolivia & 1000 & 25 & 0.9 & 0.4 & 20 & \\
\hline Amarilis & S. tuberosum & Medium & Peru & 2000 & 30 & 0.9 & 0.9 & 20 & (U. Kleinwechter, pers. comm., 2014) \\
\hline
\end{tabular}




\begin{tabular}{|c|c|c|c|c|c|c|c|c|c|}
\hline Asante & S. tuberosum & Medium & Uganda & 2000 & 26 & 0.7 & 0.9 & 21 & (U. Kleinwechter, pers. comm., 2014) \\
\hline Atlantic & S. tuberosum & Early & Peru, United States & 1000 & 25 & 0.9 & 0.6 & 17 & Hoogenboom et al. (2012) \\
\hline Bintje & S. tuberosum & Late & Europe & 1000 & 30 & 0.8 & 0.1 & 19 & \\
\hline Capiro & S. tuberosum & Late & Colombia & 1000 & 22 & 0.6 & 0.7 & 17 & Bowen et al. (1999) \\
\hline Desiree & S. tuberosum & Early & China, Peru, and Bolivia & 2000 & 25 & 0.9 & 0.6 & 16 & Hoogenboom et al. (2012) \\
\hline Dianella & S. tuberosum & Late & Denmark & 1500 & 24 & 0.6 & 0.3 & 18 & \\
\hline DTO-33 & S. tuberosum & Early & Peru & 2000 & 25 & 0.7 & 0.6 & 17 & Bowen et al. (1999) \\
\hline INIAP-Gabriela & S. tuberosum & Late & Ecuador & 2000 & 19 & 0.9 & 0.3 & 19 & Bowen et al. (1999) \\
\hline INIAP-Maria & S. tuberosum & Late & Ecuador & 2000 & 22 & 0.4 & 0.5 & 19 & Bowen et al., (1999) \\
\hline Jinguan & S. tuberosum & - & China & 2000 & 24 & 0.9 & 0.8 & 17 & \\
\hline Kaptah & S. tuberosum & Late & Denmark & 1800 & 24 & 0.6 & 0.4 & 18 & \\
\hline Kathadin & S. tuberosum & Late & United States & 2000 & 25 & 0.5 & 0.7 & 20 & Hoogenboom et al. (2012) \\
\hline Kexin 1 & S. tuberosum & - & China & 2000 & 25 & 0.9 & 0.8 & 17 & \\
\hline Kufri Bahar & S. tuberosum & Early & India & 2000 & 22 & 0.9 & 0.8 & 23 & (U. Kleinwechter, pers. comm., 2014) \\
\hline LT-1 & S. tuberosum & Early & Peru & 2000 & 25 & 0.9 & 0.8 & 17 & Hoogenboom et al. (2012) \\
\hline Luky & S. juzepczukii & Late & Bolivia & 2000 & 21 & 0.4 & 0.8 & 15 & \\
\hline Maris Piper & S. tuberosum & Late & Scotland & 2000 & 25 & 0.8 & 0.4 & 17 & Hoogenboom et al. (2012) \\
\hline Neishu 7 & S. tuberosum & - & China & 1600 & 23 & 0.7 & 0.8 & 18 & \\
\hline Posmo & S. tuberosum & Late & Denmark & 1500 & 24 & 0.6 & 0.3 & 18 & \\
\hline Ranger Russet & S. tuberosum & Late & United States & 1100 & 26 & 0.9 & 0.6 & 17 & Hoogenboom et al. (2012) \\
\hline Red Lasoda & S. tuberosum & Medium & United States & 2000 & 22 & 0.7 & 0.4 & 19 & Hoogenboom et al. (2012) \\
\hline Revolucion & S. tuberosum & Late & Peru & 2000 & 30 & 1 & 0.6 & 17 & Bowen et al. (1999) \\
\hline Russet Burbank & S. tuberosum & Late & United States & 1100 & 26 & 0.9 & 0.6 & 17 & Hoogenboom et al. (2012) \\
\hline Sarnav & S. tuberosum & Late & Peru & 1000 & 30 & 0.2 & 0.6 & 18 & \\
\hline Sava & S. tuberosum & Late & Denmark & 1300 & 24 & 0.6 & 0.4 & 21 & \\
\hline Spunta & S. tuberosum & Medium & Argentina & 1800 & 24 & 0.5 & 0.1 & 19 & Travasso et al. (1996) \\
\hline Tilva & S. tuberosum & Late & Denmark & 1500 & 24 & 0.9 & 0.3 & 18 & \\
\hline Waycha & S. andigenum & Late & Bolivia & 1200 & 23 & 0.6 & 0.3 & 15 & \\
\hline Zibaihua & S. tuberosum & - & China & 2000 & 25 & 0.9 & 0.8 & 17 & \\
\hline
\end{tabular}

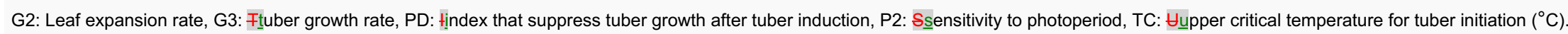

-: ANot available.

\subsection{Simulations}

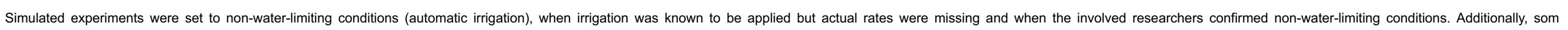




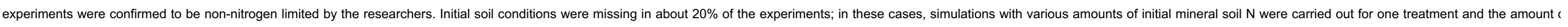

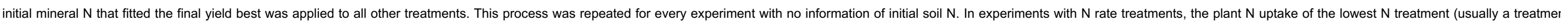

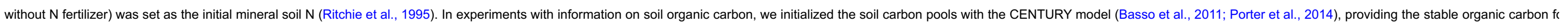
each soil layer. We assumed $82 \%$ and $90 \%$ of the total organic carbon as stable organic carbon between 0 - to $60 \mathrm{~cm}$ and below $60 \mathrm{~cm}$, respectively.

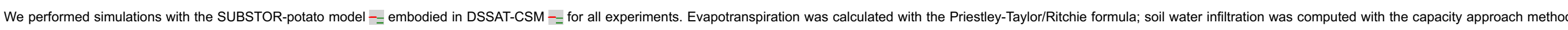
soil evaporation was estimated with the Suleiman-Ritchie method; and the dynamic of carbon and nitrogen was simulated with the CENTURY model (Hoogenboom et al., 2012).

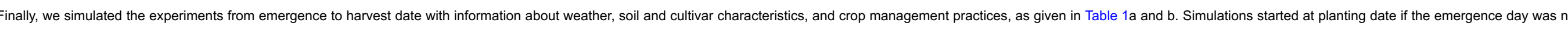
available.

\subsection{Evaluation of model performance}

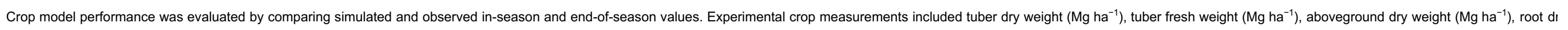

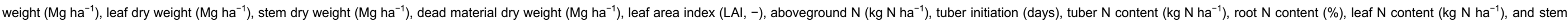
content $\left(\mathrm{kg} \mathrm{N} \mathrm{ha}^{-1}\right)$. Experimental soil measurements included soil water content $\left(\mathrm{m}^{3} \mathrm{~m}^{-3}\right)$, soil $\mathrm{NO}_{3}$

$\underline{-\mathrm{N}}(\mathrm{ppm})$, and soil $\mathrm{NH}_{4}$

$$
\text { - }
$$

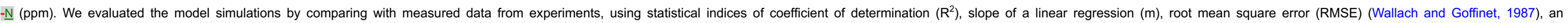

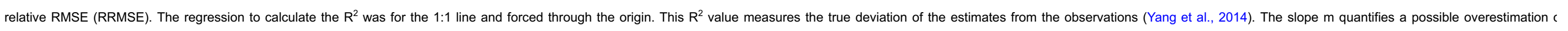
underestimation by the model. The RMSE was computed to provide a measure of the absolute magnitude of the error. All calculations and graphs were made using the R statistical software ( $R$ Core Team, 2015)

\section{Results}

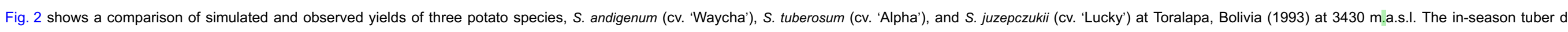

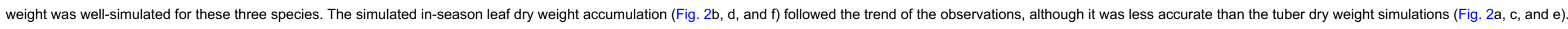




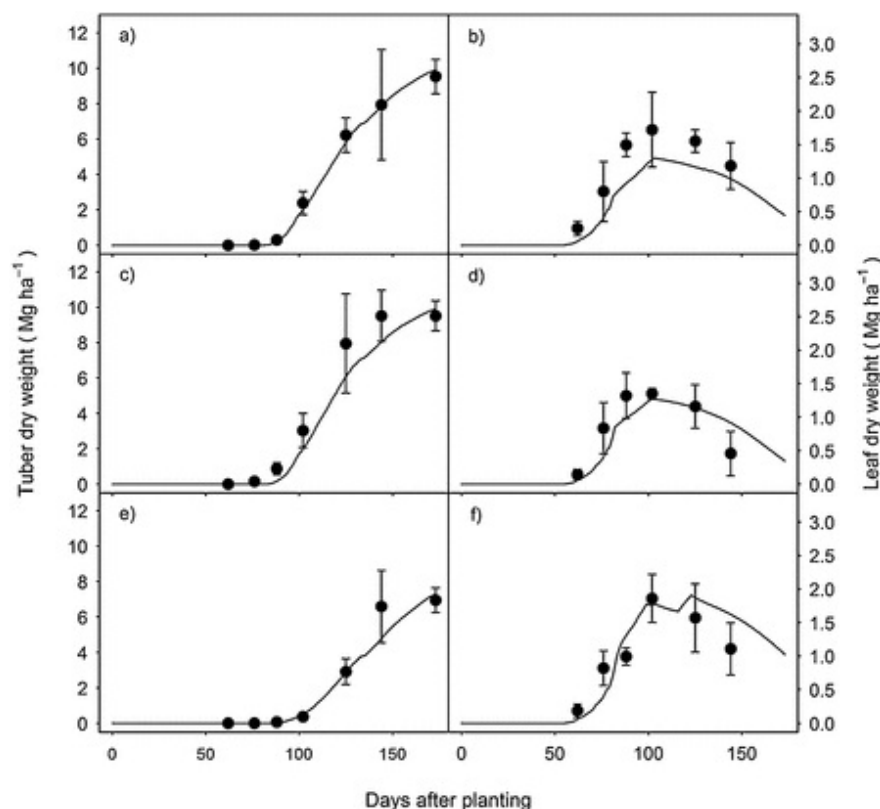

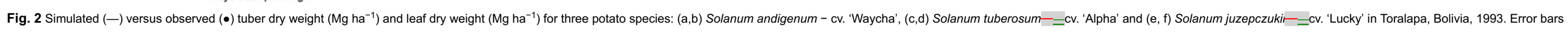
indicate standard error of measurements when available. alt-text: Fig. 2

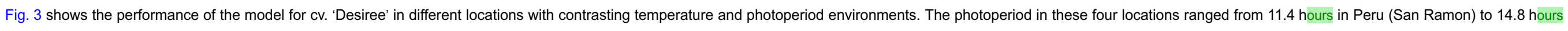

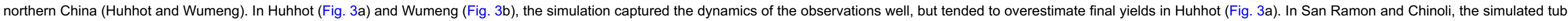

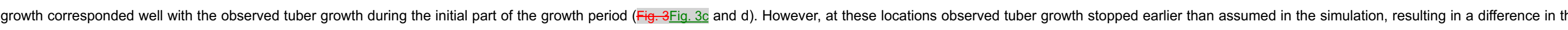
final tuber yield. 


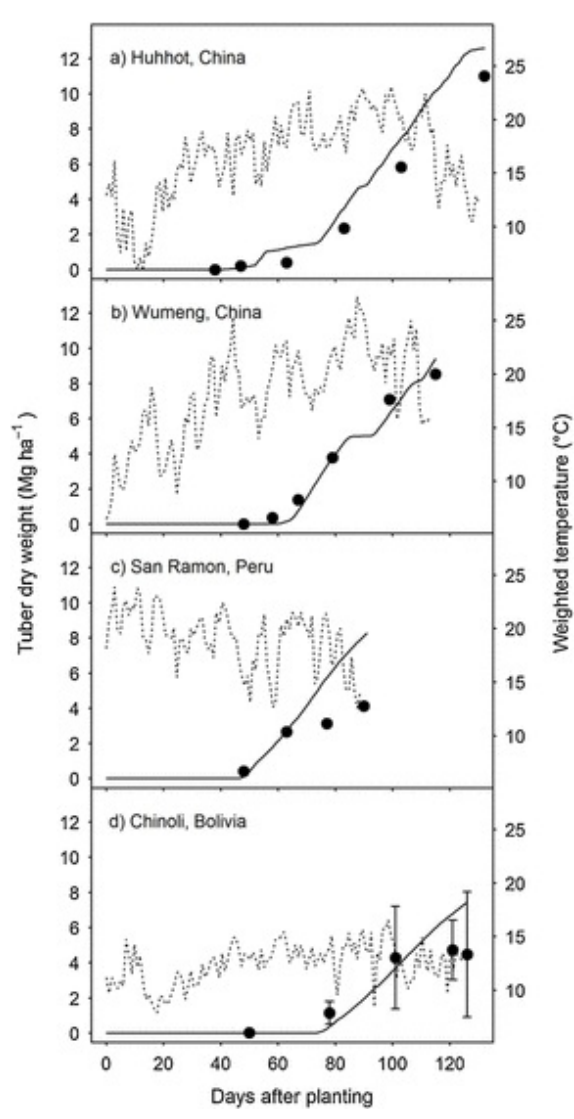

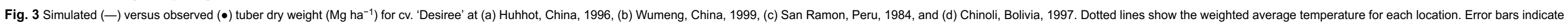
standard error of measurements when available.

\section{alt-text: Fig. 3}

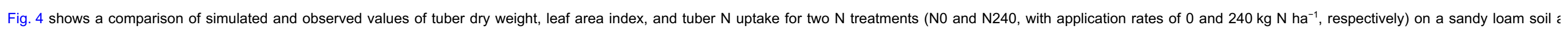

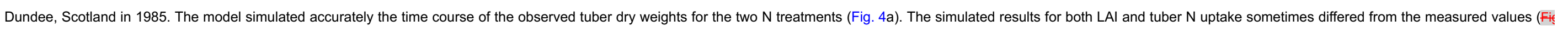

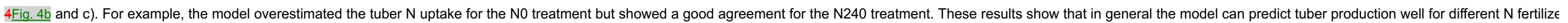
treatments, although sometimes the simulated time courses of LAl and tuber $\mathrm{N}$ uptake may differ from the observed values (Fig. 4). 


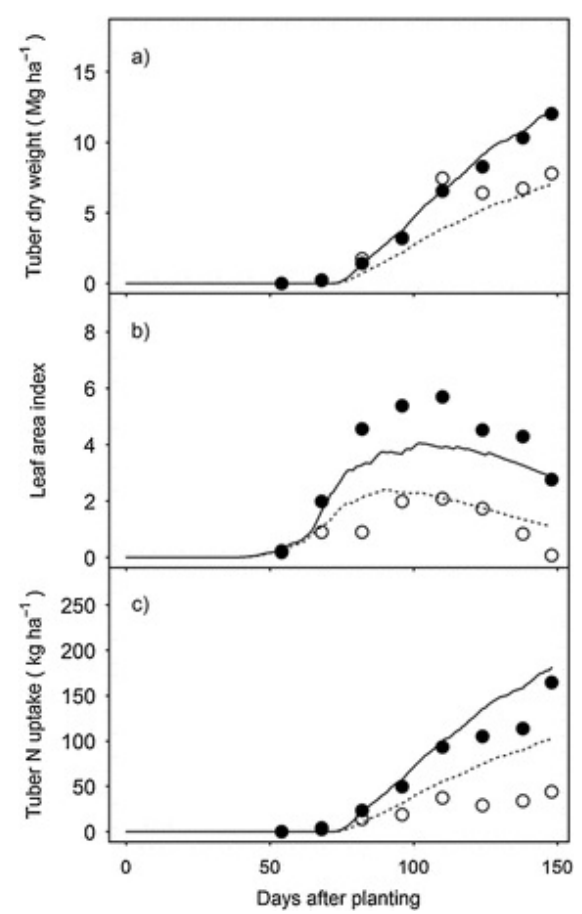

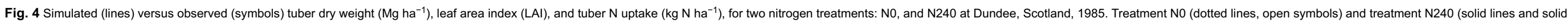
symbols).

alt-text: Fig. 4

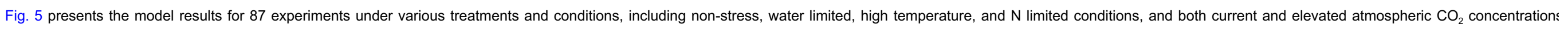

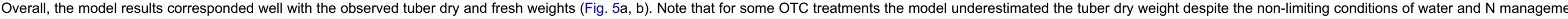

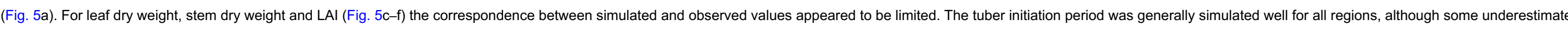
values were simulated for the high Andes (Patacamaya-3700 m. 3700 m.a.s.I) and high temperature conditions. 

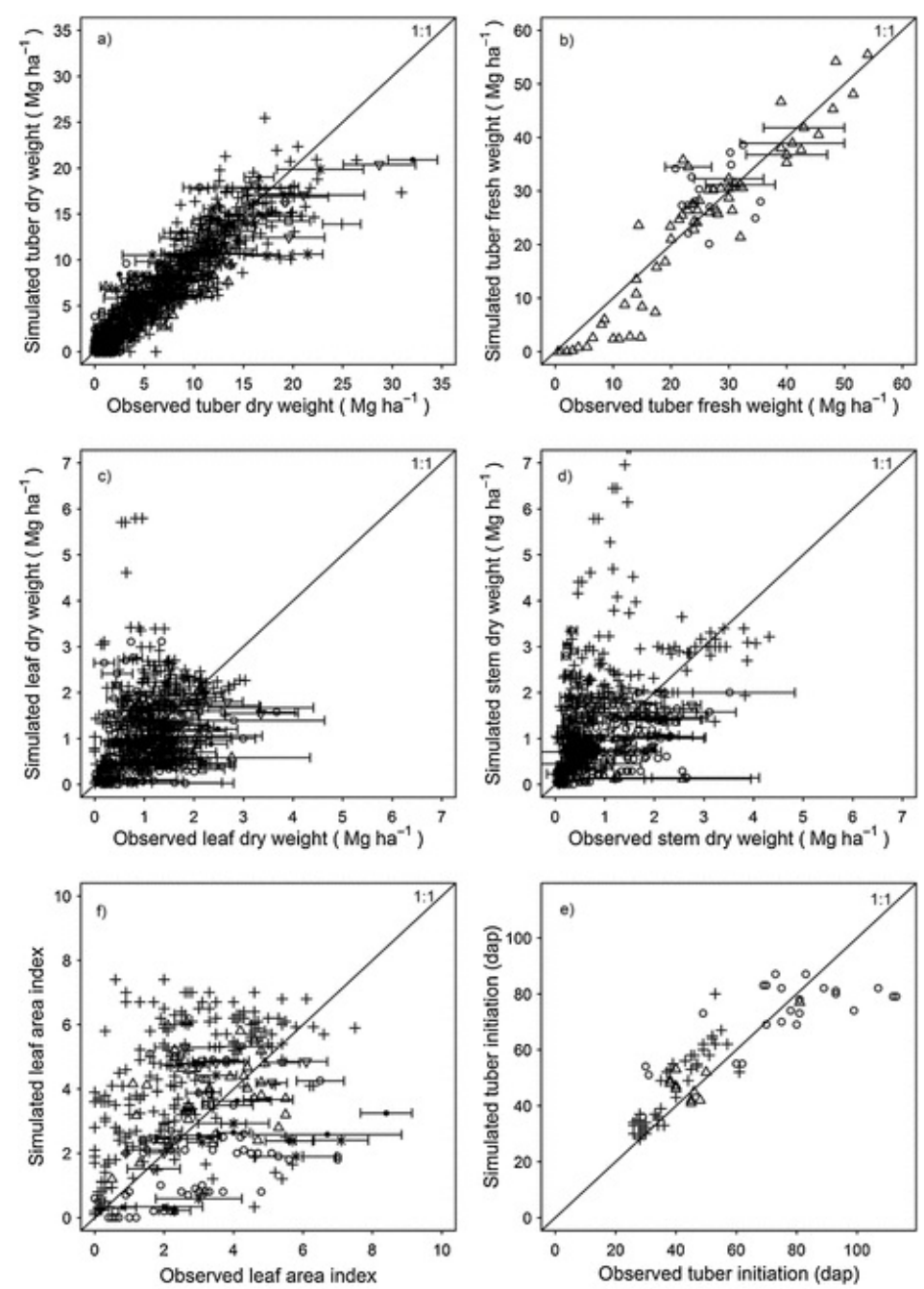

$380 \mathrm{ppm} \times 400 \mathrm{ppm} \diamond 500 \mathrm{ppm} \nabla 550 \mathrm{ppm} \cdot 680 \mathrm{ppm}$ o $700 \mathrm{ppm}$

- Tropic $\Delta$ Subtropic + Temperate

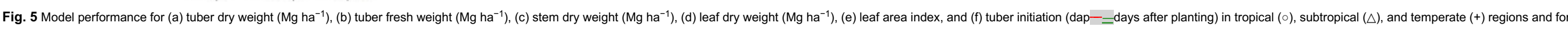
atmospheric $\mathrm{CO}_{2}$ concentrations: $380 \mathrm{ppm}\left({ }^{\star}\right), 400 \mathrm{ppm}(\times), 500 \mathrm{ppm}(\diamond), 550 \mathrm{ppm}(\nabla), 680 \mathrm{ppm}(\bullet), 700 \mathrm{ppm}(\square)$, for FACE and OTC experiments. Error bars indicate standard error of measurements when available. alt-text: Fig. 5

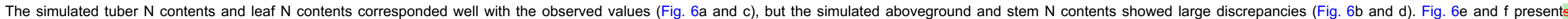

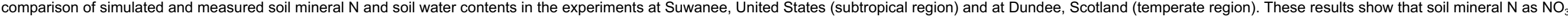

\section{Nand NH4-}

$\underline{N}$ (not shown in figure) were poorly simulated; additionally, the observed data had large measurement errors. The soil water contents were moderately well simulated for Suwannee but underestimated for Dundee. 

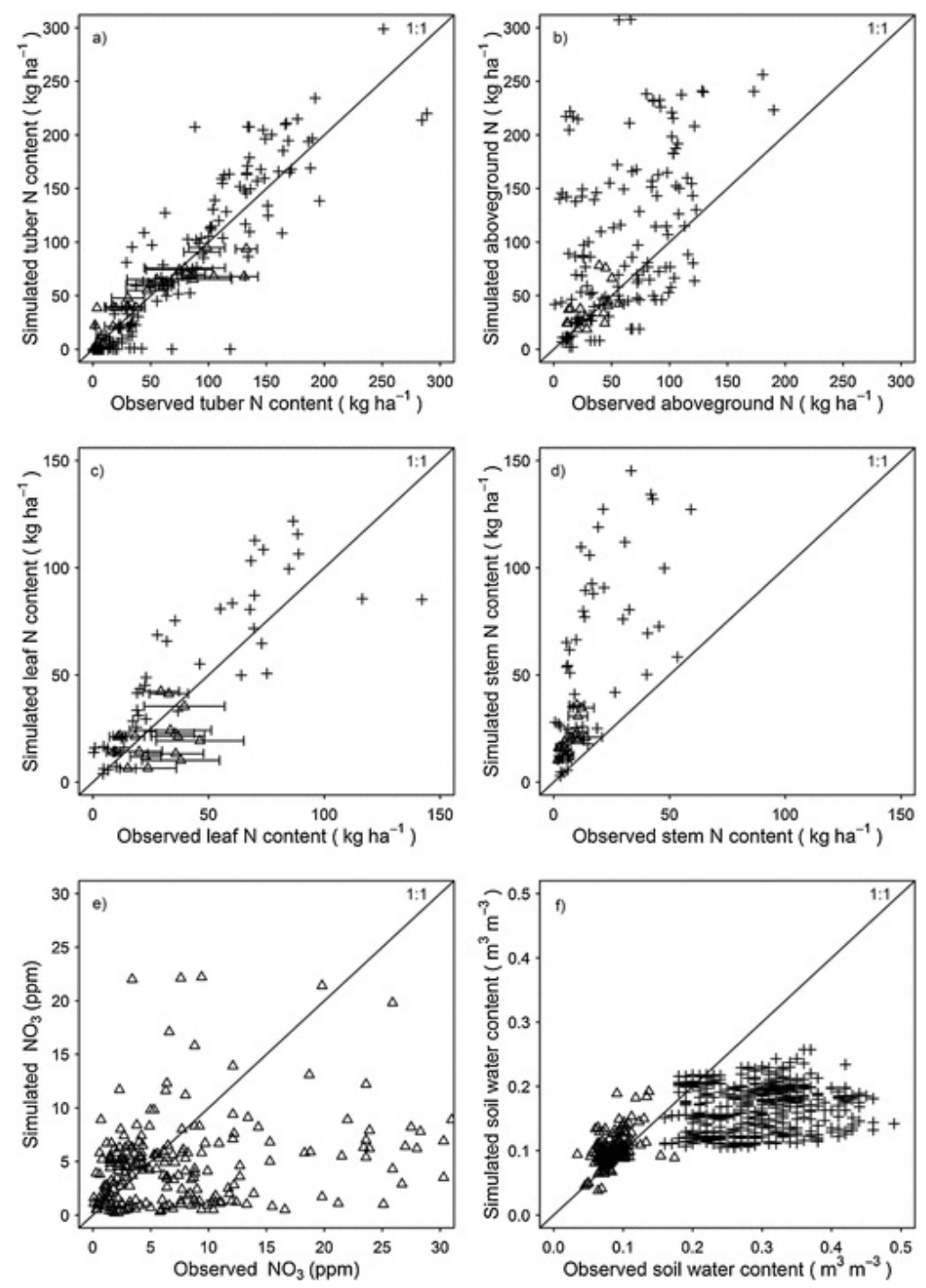

Fig. 6 Model performance of (a) tuber $\mathrm{N}$ uptake $\left(\mathrm{kg} \mathrm{N} \mathrm{ha}^{-1}\right)$, (b) aboveground $\mathrm{N}$ uptake $\left(\mathrm{kg} \mathrm{N} \mathrm{ha}^{-1}\right)$, (c) leaf $\mathrm{N}$ uptake $\left(\mathrm{kg} \mathrm{N} \mathrm{ha}^{-1}\right)$, (d) stem N uptake $\left(\mathrm{kg} \mathrm{N} \mathrm{ha}^{-1}\right)$, (e) soil mineral $\mathrm{N}$ as a NO${ }_{3}$

$-\mathrm{N}(\mathrm{ppm})$, and (f) soil water content $\left(\mathrm{m}^{3} \mathrm{~m}^{-3}\right)$ for subtropical $(\triangle)$, and temperate $(+)$ regions. Error bars indicate the standard deviation of measurements when available. For panel (e) and (f) error bars were available but not shown. alt-text: Fig. 6

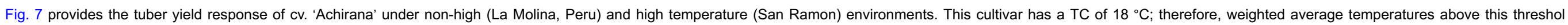

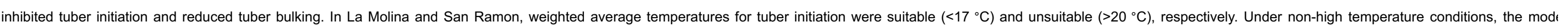

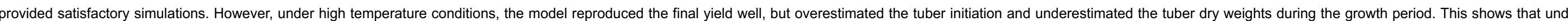
high temperature conditions throughout the growing season, SUBSTOR-potato failed to simulate the observed growth dynamics well. 


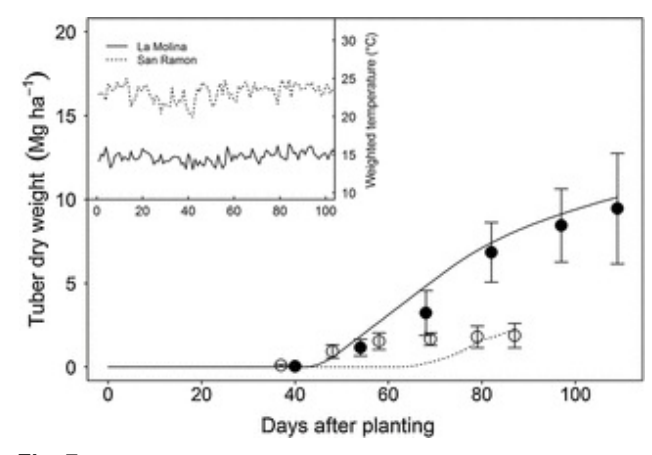

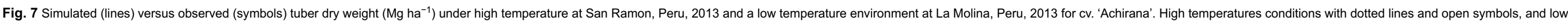
temperatures conditions with solid lines and solid symbols. Error bars indicate standard error of measurements when available. Weighted average temperature for both locations is embedded in the graphic.

alt-text: Fig. 7

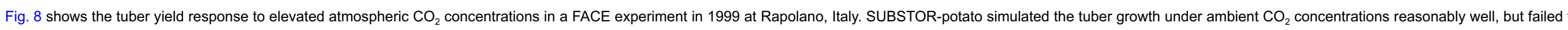
simulate the large positive observed yield response to elevated atmospheric $\mathrm{CO}_{2}$.

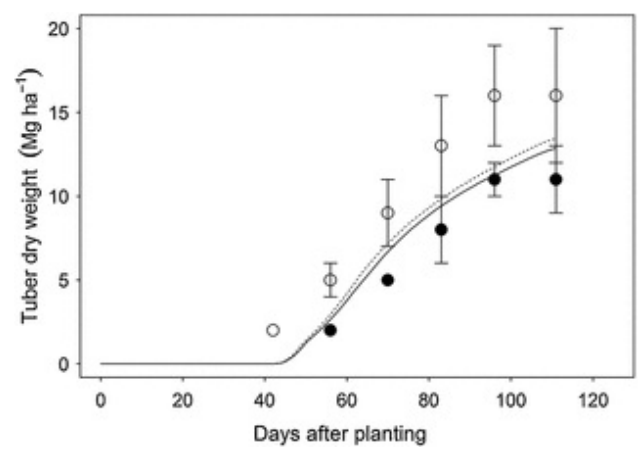

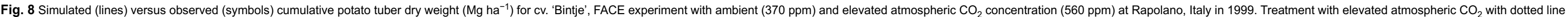
and open symbols, and treatment with ambient $\mathrm{CO}_{2}$ with solid lines and solid symbols. Error bars indicate standard error of measurements when available.

\section{alt-text: Fig. 8}

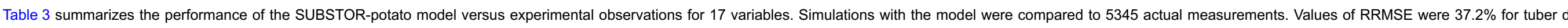
weight, $21.0 \%$ for tuber fresh weight, $22.6 \%$ for tuber initiation, $40.4 \%$ for tuber $\mathrm{N}$ uptake, and $52.3 \%$ for leaf $\mathrm{N}$ uptake. RRMSE for stem $\mathrm{N}$ content, aboveground $\mathrm{N}$ content, and soil $\mathrm{NO}_{3}$ and $\mathrm{NH}_{4}$ were $>80 \%$.

Table 3 Summary of model performance of SUBSTOR-potato modelling for all experiments (see Table 1).

alt-text: Table 3

\begin{tabular}{|c|c|c|c|c|c|c|}
\hline Variable & Number of paired data & Observed range & $\mathrm{R}^{2}$ & Slope & RMSEa & RRMSEb \\
\hline Tuber dry weight (Mg ha-1) & 946 & $0.00 \_\_32.08$ & 0.93 & 1.01 & 2.12 & 37.20 \\
\hline Tuber fresh weight (Mg ha-1) & 79 & $0.50-54$ & 0.97 & 0.96 & 5.23 & 21.04 \\
\hline Aboveground dry weight $\left(\mathrm{Mg} \mathrm{ha}^{-1}\right)$ & 758 & $0.03-9.75$ & 0.69 & 0.64 & 1.93 & 85.33 \\
\hline Roots dry weight(Mg ha-1) & 213 & $0.02-1.08$ & 0.63 & 0.45 & 0.34 & 253.07 \\
\hline
\end{tabular}




\begin{tabular}{|c|c|c|c|c|c|c|}
\hline Leaf dry weight (Mg ha-1-1) & 504 & $0.01-3.67$ & 0.61 & 0.64 & 0.97 & 90.34 \\
\hline Stem dry weight (Mg ha-1) & 471 & $0.02-4.32$ & 0.51 & 0.48 & 1.32 & 141.42 \\
\hline Dead dry weight (Mg ha-1) & 57 & 0 00d_2.56 & 0.26 & 0.79 & 0.62 & 151.42 \\
\hline LAI & 378 & $0.01-8.39$ & 0.70 & 0.67 & 2.24 & 81.95 \\
\hline Tuber initiation (dap) & 116 & $26.00-113$ & 0.96 & 0.93 & 10.54 & 22.65 \\
\hline Tuber N (kg ha-1) & 184 & $0.10-318.20$ & 0.92 & 0.90 & 44.78 & 40.40 \\
\hline Aboveground N (kg ha-1) & 160 & $76.48-132.72$ & 0.78 & 0.65 & 76.48 & 86.23 \\
\hline Roots N (kg ha-1) & 96 & $0.63-8.19$ & 0.60 & 0.37 & 6.95 & 153.83 \\
\hline Leaf $N(k$ ha-1) & 64 & $21.48-52.32$ & 0.86 & 0.89 & 21.48 & 52.31 \\
\hline Stem N (kg ha-1) & 64 & $0.62-59.25$ & 0.74 & 0.28 & 47.18 & 309.57 \\
\hline Soil water content $\left(\mathrm{m}^{3} \mathrm{~m}^{-3}\right)$ & 891 & $0.03-0.49$ & 0.89 & 0.89 & 1.55 & 62.08 \\
\hline $\mathrm{NO}_{3}-\underline{-N}(\mathrm{ppm})$ & 240 & $0.10-58.10$ & 0.42 & 1.35 & 9.82 & 95.45 \\
\hline $\mathrm{NH}_{4}-\underline{-N}(\mathrm{ppm})$ & 240 & $0.60-185.60$ & 0.05 & 1.66 & 19.42 & 140.09 \\
\hline
\end{tabular}

a Root mean square error $\left(\mathrm{Mg} \mathrm{ha}^{-1}\right)$.

${ }^{\mathrm{b}}$ Relative root mean square error $(\%)$.

c 0.00002 .

d 0.00002 .

\section{Discussion}

\subsection{Cultivars of the developed world}

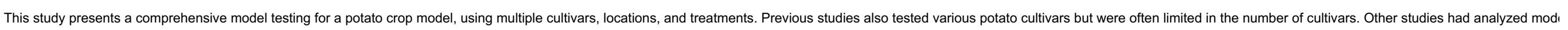

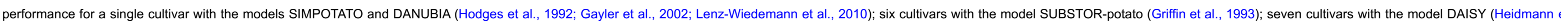

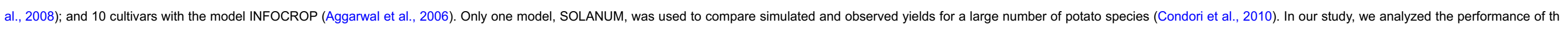
SUBSTOR-potato model for three potato species and 32 potato cultivars. The results indicated that the SUBSTOR-potato model can in general reproduce the tuber and leaf development for different potato species and cultivars.

\subsection{Cultivar use across climatic regions}

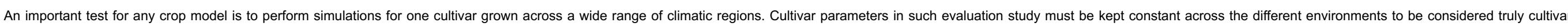

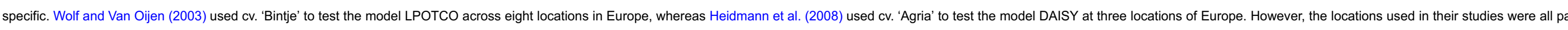

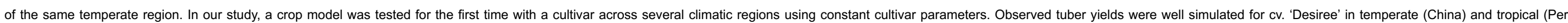

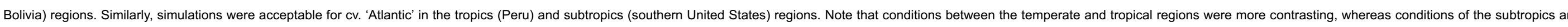

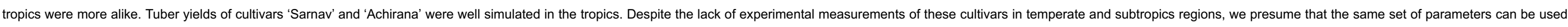

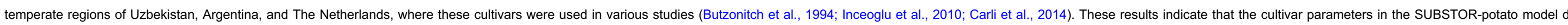
represent cultivar-specific characteristics and support the general functionality and transferability of this model. 


\section{$5.3 \mathrm{~N}$ response}

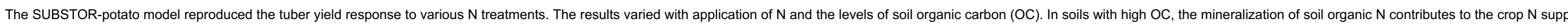

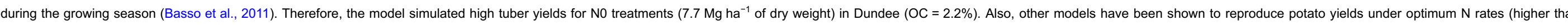

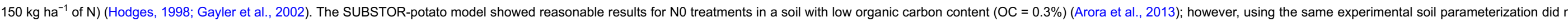

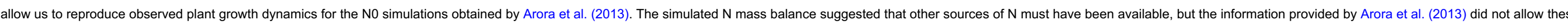

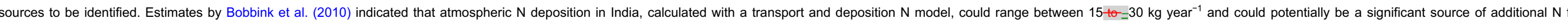

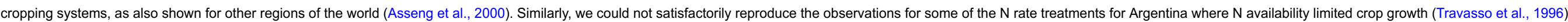

\subsection{Simulation of tuber yield and other variables}

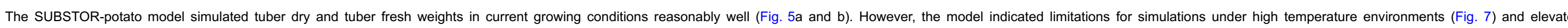

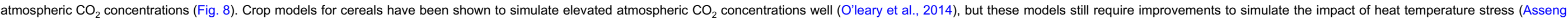
al., 2015) and interactions of $\mathrm{CO}_{2}$ with high temperatures (Asseng et al., 2013).

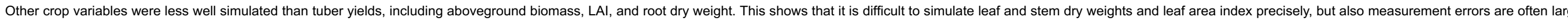

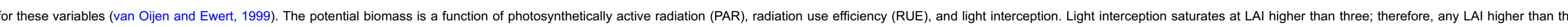
value will have the same impact on potential biomass.

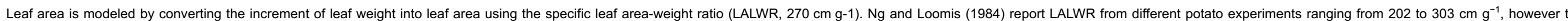

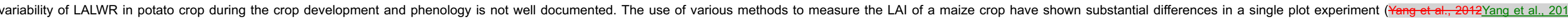

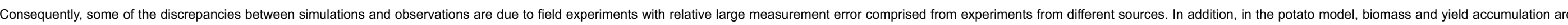

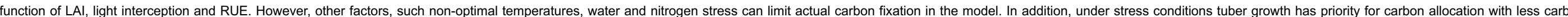
allocation to leaves and stems. Hence, while LAl is important for light interception, it is often not the limiting factor for yield.

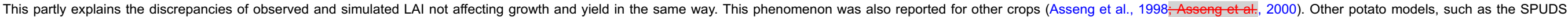

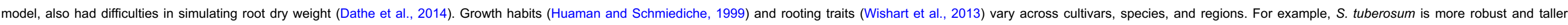

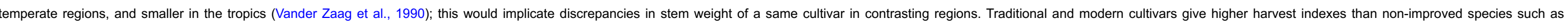

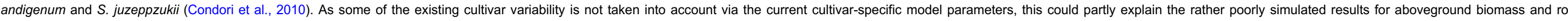
weights. In addition, the quality of root measurements in potatoes could be a factor in some model observation discrepancies (Ahmadi et al., 2014).

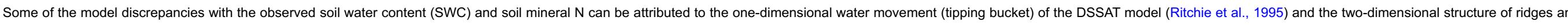
valleys in potato fields.

\subsection{Overestimation at the end of the growing season}

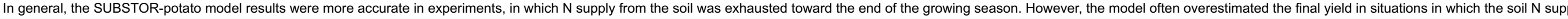

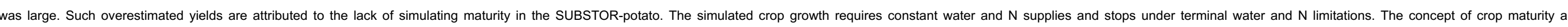

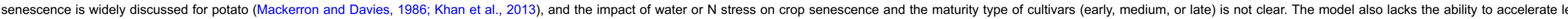

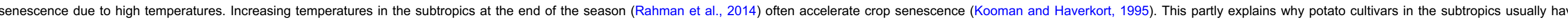
shorter growing periods (Santhosh et al., 2014).

\subsection{Simulations under high temperatures}

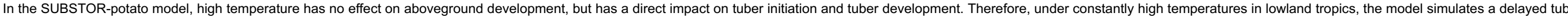




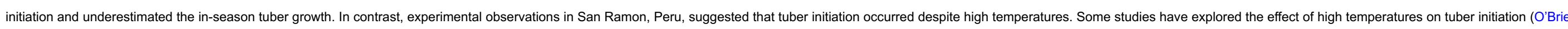

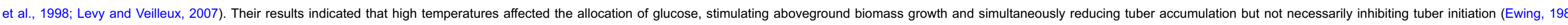

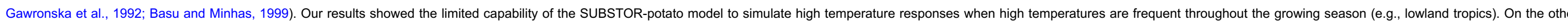

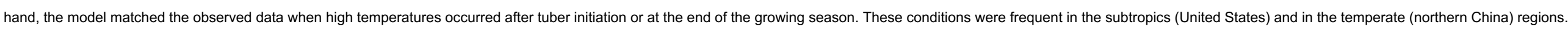

\section{$5.7 \mathrm{CO}_{2}$ effect}

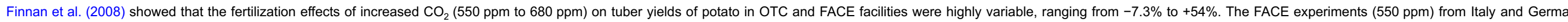

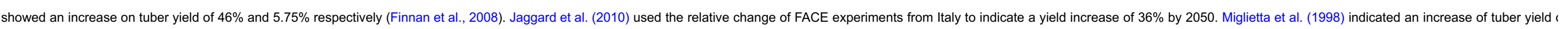

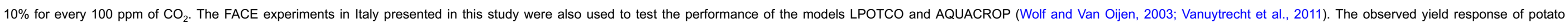

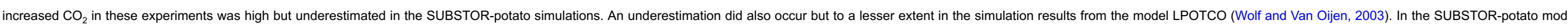

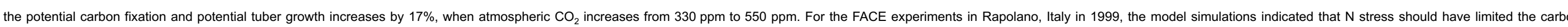

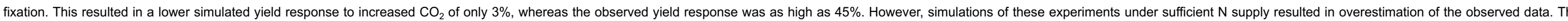

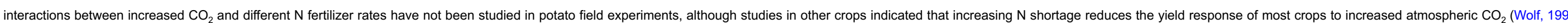

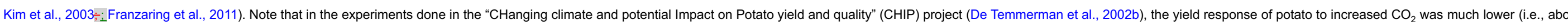
$+20 \%$ to $+30 \%$ if the $\mathrm{CO}_{2}$ concentration increased from 380 to $550 \mathrm{ppm}$ ) than that observed in the FACE experiments in Rapolano (De Temmerman et al., 2002b; Wolf and Van Oijen, 2003).

\section{Conclusion}

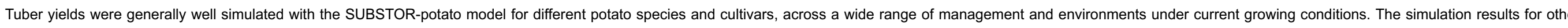

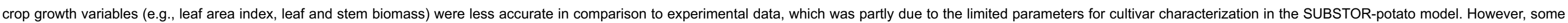

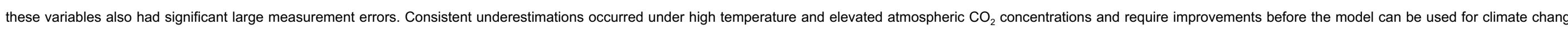
impact assessments.

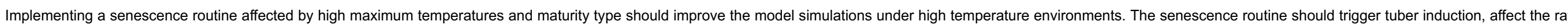

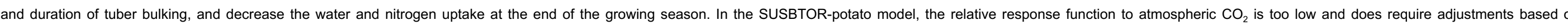
experimental data.Uncited referencesAlbert (2002), Berloo R. v. Hutten et al. (2007), ZZZZ (2016), Lutaladio and Castaidi (2009), Lutz (2010).

\section{Acknowledgements}

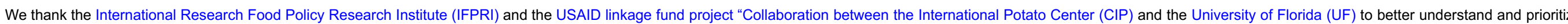
climate change adaptation needs for food security in the Andes".

\section{References}

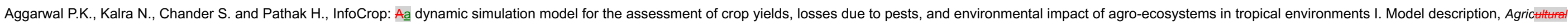
Systems. Syst. 89, 2006, 1-25.

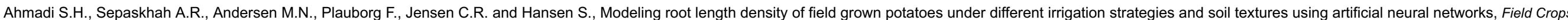
Resareh. 162, 2014, 99-107.

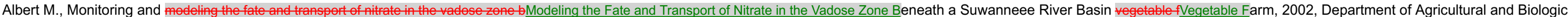
Engineering; University of Florida, Gainesville, Florida, pp. 1-179.

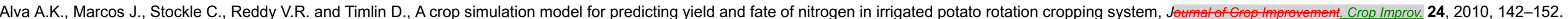




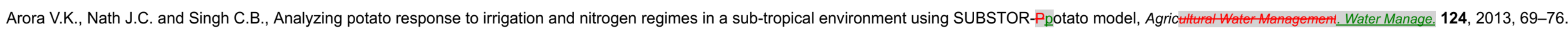

Asseng S., Keating B.A., Fillery I.R.P., Gregory P.J., Bowden J.W., Turner N.C., Palta J.A. and Abrecht D.G., Performance of the APSIM-wheat model in Wwestern Australia, Field Crops Researeh 57, 1998, 163-179.

Asseng S., van Keulen H. and Stol W., Performance and application of the APSIM Nwheat model in the Netherlands, Eur

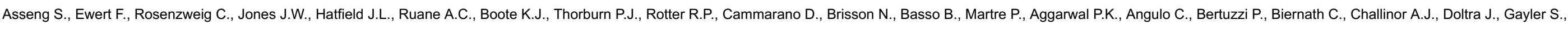

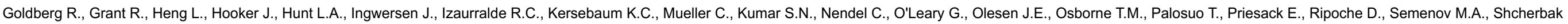

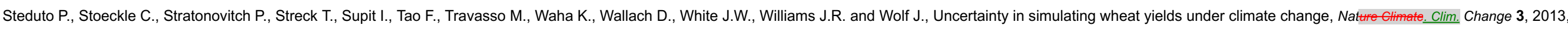
$827-832$.

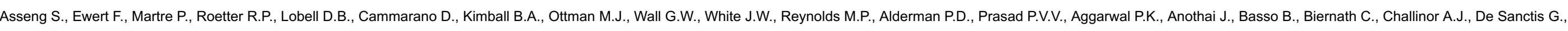

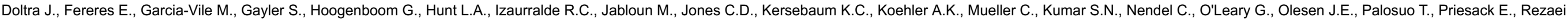

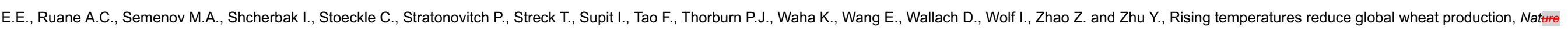
Elimate. Clim. Change 5, 2015, 143-147.

Bach A. and Nielsen S., Vækstanalyse i kartofler 1981-_-83, Planteavl 89, 1985, 215-224.

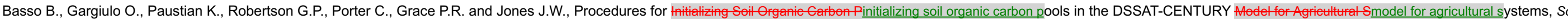
Scien Soc. Am. J. 75, 2011, 69-78.

Basu P.S. and Minhas J.S., Tuberization at high temperatures in different potato genotypes, , Indian Potato Assoc. 26, 1999, 19-22.

Berloo R.v., Hutten R.C.B., Eck H.J.V., Visser R.G.F., van Berloo R. and van Eck H.J., An online potato pedigree database resource, Potato Research_ 50, 2007, 45-57.

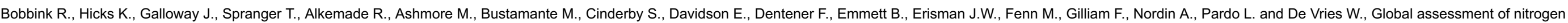

deposition effects on terrestrial plant diversity: a synthesis, Ecolegieal Applications. Appl. 20, 2010, 30-59.

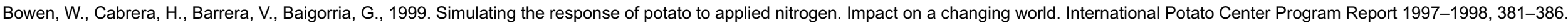

Butzonitch, I.P., Colavita, M.L., Lucarini, O., 1994. Potato virus M in Argentina. Boletin Tecnico-Estacion Experimental Agropecuaria, Balcarce, 11 pp.-11 pp.

CIP, Annual ÆReport 1992, 1992, International Potato Center; Lima-Peru.

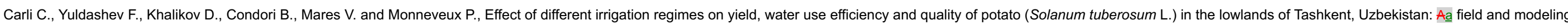
perspective, Field Crops Researeh, 163, 2014, 90-99.

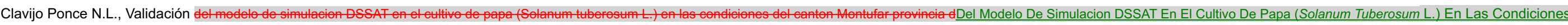
Del Canton Montufar Provincia Del Carchi, 1999, Escuela de ingenieria agronómica, Escuela superior politécnica de Chimborazo; Riobamba, Ecuador, p.80.

Condori B., Hijmans R.J., Quiroz R. and Ledent J.F., Quantifying the expression of potato genetic diversity in the high Andes through growth analysis and modeling, Field Crops Researeh. 119, $2010,135-144$.

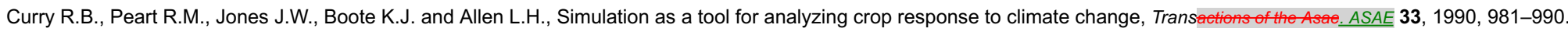

Dathe A., Fleisher D.H., Timlin D.J., Fisher J.K. and Reddy V.R., Modeling potato root growth and water uptake under water stress conditions, Agricutturat and Forest Aleteory. Forest Meteorol. 194, 2014, 37-49.

De Temmerman L., Hacour A. and Guns M., Changing climate and potential impacts on potato yield and quality 'CHIPI: introduction, aims and methodology, Eurepean dournal of Agromy. J. Agron. 17, $2002 a, 233-242$.

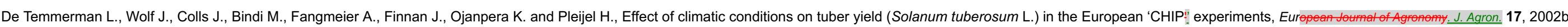
243-255. 
Edlefsen O., Styring af kvælstof $\mathrm{i}$ vandede kartofler, Science, 1991, T.D.I.o.A.

Ewing E.E., Heat-stress and the tuberization stimulus, A Potato J. 58, 1981, 31-49.

FAO, FAOSTAT, $2010 \mathrm{http}: / /$ faostat.fao.org/.

Finnan J.M., Donnelly A., Jones M.B. and Burkec J.I., The effect of elevated levels of carbon dioxide on potato crops,

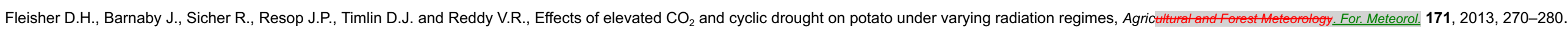

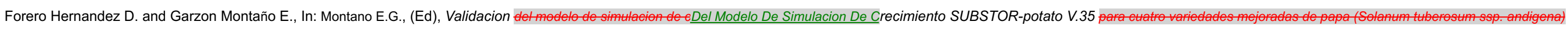

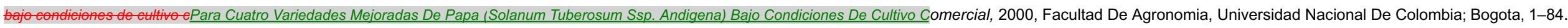

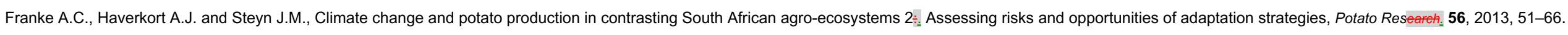

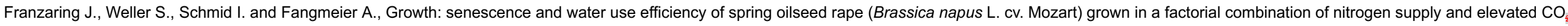
Environ. Exp. Bot. 72, 2011, 284-296.

Gao J.L., Liu K.L., Zhang B.L. and Ren Y.Z., Accumulation and distribution of dry matter in potato, China Potato 17, 2003, 209-212.

Gao J.L., Liu K.L., Sheng J.H., Ren K., Wen X.J., Sui Q.J. and Jiang B., Dry matter accumulation and distribution of potato under dry farming, Potato China 18, $2004,9-15$.

Gawronska H., Thornton M.K. and Dwelle R.B., Influence of heat-stress on dry-matter production and photoassimilate partitioning by 4 potato clones, Amerieatate jotat. 69 , 1992, 653-665.

Gayler S., Wang E., Priesack E., Schaaf T. and Maidl F.X., Modeling biomass growth, N-uptake and phenological development of potato crop, Geoderma 105, 2002, 367-383.

Gobin A., Modelling climate impacts on crop yields in Belgium, Climate Research. Res. 44, 2010, 55-68.

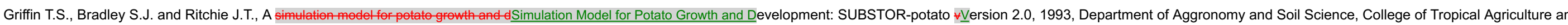
Human Resources, University of Hawai; Honolulu, p. 29.

Haverkort A. and Top J., The potato ontology: delimitation of the domain, modelling concepts, and prospects of performance, Potato Researeh_54, $2011,119-136$.

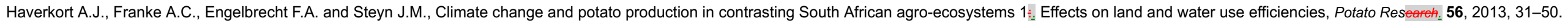

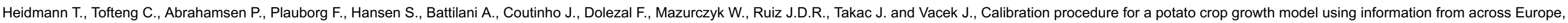
Ecologicalling. Modell. 211, 2008, 209-223.

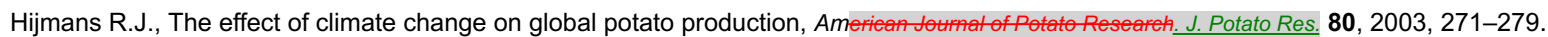

Hodges T., Johnson S.L. and Johnson B.S., A modular structure for crop simulation models: timplemented in the SIMPOTATO model, Agronomy Journat.J. 84, 1992, 911-915.

Hodges T., Water and nitrogen applications for potato: Eçommercial and experimental rates compared to a simulation model, Jeunal of Surtainable Agrieutture. Sustainable Agric. 13, 1998, 79-90.

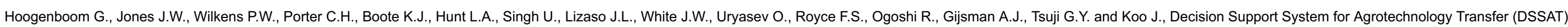
Version 4.5 [CD-ROM], 2012, University of Hawaii; Honolulu, Hawaii.

Huaman Z. and Schmiediche P., The potato genetic resources held in trust by the International Potato Center (CIP) in Peru, Potato Researeh_. 42, 1999, 413-426.

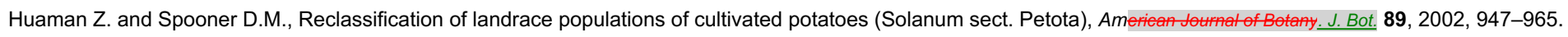

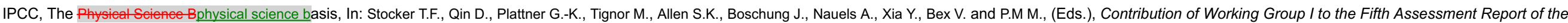


Intergovernmental Panel on Climate Change, 2013, Cambridge University Press Cambridge; United Kingdom and New York, NY, USA, p. 1535.

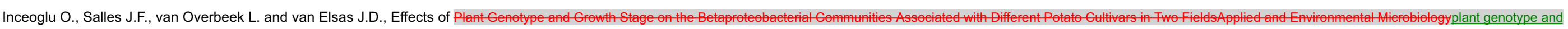

growth stage on the betaproteobacterial communities associated with different potato cultivars in two fields, Appl. Environ. Microbiol. 76, 2010, 3675-3684.

Jørgensen V. and Edlefsen O., Vandforsyningens indflydelse på udbytte og kvalitet af industrikartofler, Planteavl 91, 1987, 329-347.

Jørgensen V., Vandforsyningens indflydelse på udbytte og kvalitet af kartofler, Planteavl 88, 1984, 453-468.

Jaggard K.W., Qi A. and Ober E.S., Possible changes to arable crop yields by 2050, Philospheal Trans 2010 , 2835-2851.

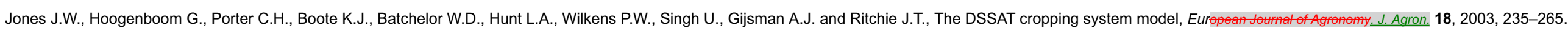

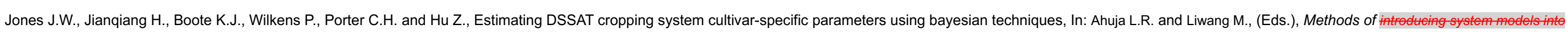

agrieutturatfIntroducing System Models into Agricultural Research, 2011, American Society of Agronomy, Crop Science Society of America, Soil Science Society of America Madison; WI, USA.

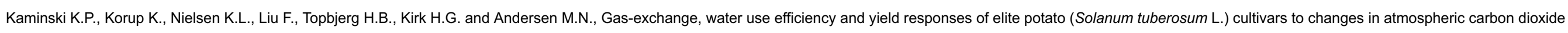
concentration, temperature and relative humidity, Agricultural and Forest Meteorology. Forest Meteorol. 187, 2014, $36-45$.

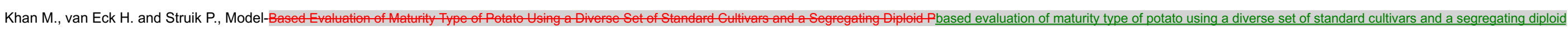
population, Potato Research. 56, 2013, 127-146.

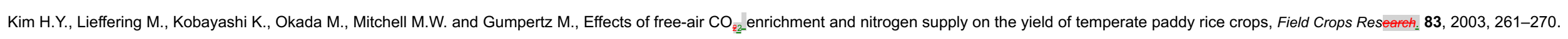

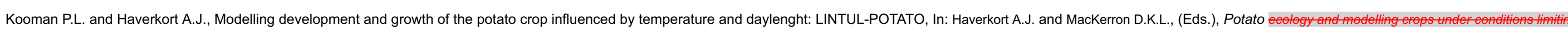
gEcology and Modelling Crops Under Conditions Limiting Growth, 1995, Kluwer Academic Publisher; Wageningen, The Netherlands, 41-59.

Lenz-Wiedemann V.I.S., Klar C.W. and Schneider K., Development and test of a crop growth model for application within a global change decision support system, Ecolegical Allling. Modell. 221, 2010 , 314-329.

Levy D. and Veilleux R.E., Adaptation of potato to high temperatures and salinity-AreviewAmerican dournal of Potato Researcha review, Am. J. Potato Res. 84, 2007, 487-506.

Liu K.L., Gao J.L., Ren K., Sheng J.H., Sui Q.J. and Jiang B., Nitrogen absorption, accumulation and distribution of potato under dry farming, Potato China 17, 2003a, 321-325.

Liu K.L., Gao J.L., Sun H.Z. and Sheng J.H., The dynamic of sink structure in potato, China Potato 17, 2003b, 267-272.

Lutaladio N. and Castaidi L., Potato: Fthe hidden treasure, 2009.

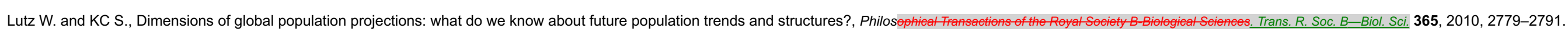

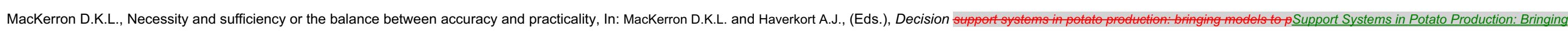
Models to Practice, 2004, Wageningen academic publishers; The Netherlands, 213-223.

Mackerron D.K.L. and Davies H.V., Markers for maturity and senescence in the potato crop, Potato Reseh_ 29, 1986, 427-436.

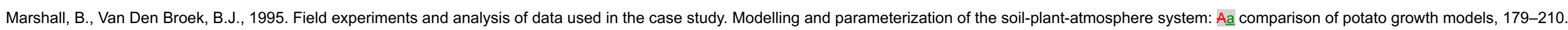

Miglietta F., Magliulo V., Bindi M., Cerio L., Vaccari F., Loduca V. and Peressotti A., Free air CO(2) enrichment of potato (Solanum tuberosum L.): development growth and yield, Global Change Biolegy: 4, $1998,163-172$.

Monfreda C., Ramankutty N. and Foley J.A., Farming the planet: 2. Geographic distribution of crop areas, yields, physiological types, and net primary production in the year 2000, Global Biogeochemieat_ Cycles 22, $2008,19$.

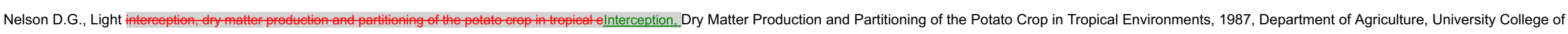
Wales, 1-135. 


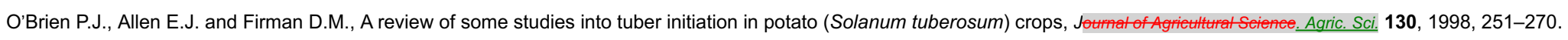

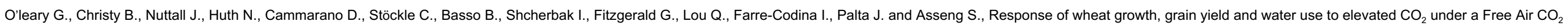
Enrichment (FACE) experiment and modelling in a semi-arid environment, Global Change Biofyly. 2014.

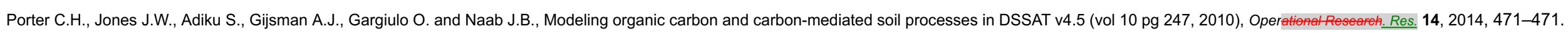

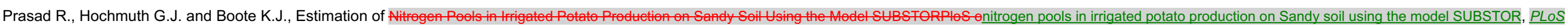

One 10, 2015, e0117891-e0117891.

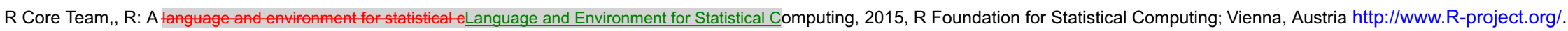

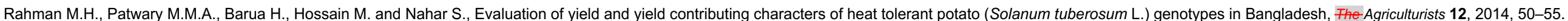

Raymundo R., Asseng S., Cammarano D. and Quiroz R., Potato sweet potato, and yam models for climate change: Aa review, Field Crops Researeh. 166, $2014,173-185$.

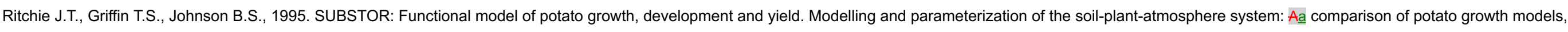
$401-435$.

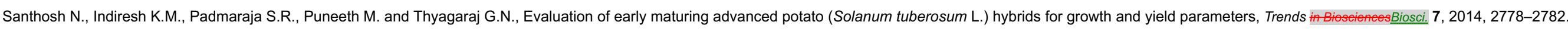
Saue T. and Kadaja J., Possible effects of climate change on potato crops in Estonia, Boreal Environ 2011, 203-217.

Stockle C.O., Nelson R.L., Higgins S., Brunner J., Grove G., Boydston R., Whiting M. and Kruger C., Assessment of climate change impact on eastern Washington agriculture, Climatie. Change 102, $2010,77-102$.

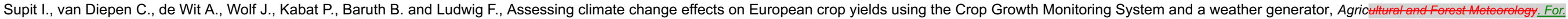
Meteorol. 164, 2012, 96-111.

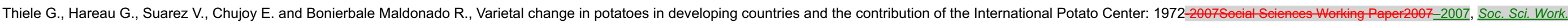
Pap. 2007, p. 46 .

Travasso M.I., Caldiz D.O. and Saluzzo J.A., Yield prediction using the SUBSTOR-potato model under Argentinian conditions, Potato Researeh_. 39, 1996, 305-312.

Trebejo I. and Midmore D.J., Effect of water stress on potato growth, yield and water use in a hot and a cool tropical climate, The Journalof Agrieuttural seienee 2 . Agric. Sci. 114, 1990, 321-334.

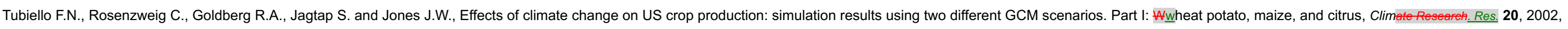
$259-270$.

Vander Zaag P., Demagante A.L. and Ewing E.E., Influence of plant spacing on potato (Solanum tuberosum L.) morphology: growth and yield under two contrasting environments, Potato Researeh. 33, 1990, 313-323.

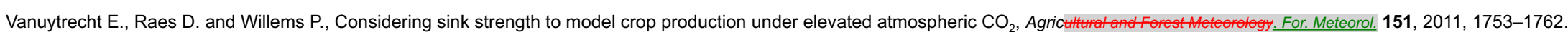

Wallach D. and Goffinet B., Mean square error of prediction in models for studying ecological and agronomics sytems, Biomestrics 43, 1987, 561-573.

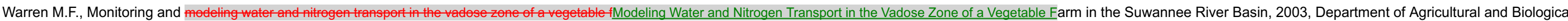
Engineering, University of Florida; Gainesville, Florida, pp.1-233.

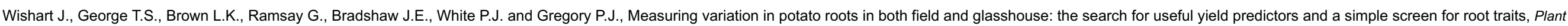
Soil 368, 2013, 231-249.

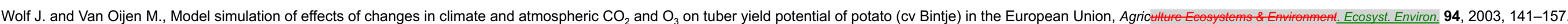


Wolf J., Effects of nutrient supply (NPK) on spring wheat response to elevated atmospheric $\mathrm{CO}_{2}$, Plant and Soil 185, 1996, 113-123.

Yang J.M., Yang J.Y., Liu S. and Hoogenboom G., An evaluation of the statistical methods for testing the performance of crop models with observed data, Agrictttural Systems. Syst. 127, 2014, 81-89.

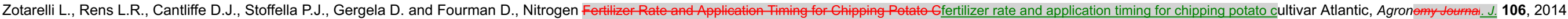
2215-2226.

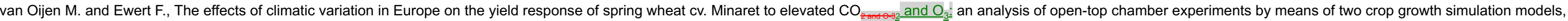
Eurany. J. Agron. 10, 1999, 249-264.

Highlights

- The SUBSTOR-=potato model was tested with 87 experiments and 204 treatments, including 32 cultivars and three potato species.

- The model-observation comparison showed that the SUBSTOR-potato model can in general simulate tuber yields across contrasting environments.

- However, the SUBSTOR-potato model cannot accurately simulate tuber yield under elevated $\mathrm{CO}_{2}$ concentrations and high temperatures which needs to be improved.

\section{Queries and Answers}

Query: The author names have been tagged as given names and surnames (surnames are highlighted in teal color). Please confirm if they have been identified correctly

Answer: Everything is correct.

Query: Please check the edits made in affiliations "a", "g" and "j" and correct if necessary.

Answer: Correct.

Query: Highlights must be provided as 3-5 bullet points, each bullet point having a maximum of 125 characters. Please rephrase the following highlights or provide new highlights.

Answer: A file is attached.

Query: Please check the keywords and correct if necessary.

Answer: OK.

Query: Please check the hierarchy of the section headings.

Answer: Correct.

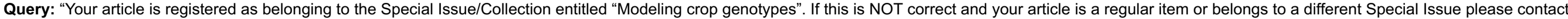
j.miranda@elsevier.com immediately prior to returning your corrections."

Answer: belongs to $\mathrm{SI}$ is correct.

Query: The citation "Ritchie, 1998" has been changed to "Ritchie et al., 1995" to match the reference list. Please check and correct if necessary.

Answer: OK

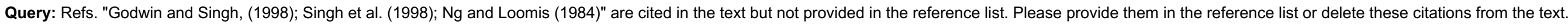
Answer: Please keep only $\mathrm{Ng}$ and Loomis (1984): 


\section{Ng, E., Loomis, R.S., 1984. Simulation of growth and yield of the potato crop. Centre for agricultural publishing and documentation, Wageningen, The Netherlands.}

Remove: Sing et al. 1998 and Godwin and Singh 1998

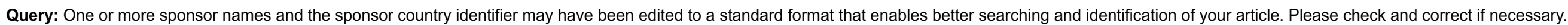
Answer: OK.

Query: Please check the author names in Ref. "Finnan et al., 2008" and correct if necessary.

Answer: Please replace with:

Finnan, J.M., Donnelly, A., Jones, M.B., Burke, J.I., 2008. The effect of elevated levels of carbon dioxide on potato crops. Journal of Crop Improvement 13 , 91-111.

Query: Please supply the name of the city of publication.

Answer: sorry, can't find the city name.

Query: Please check the presentation of all the Tables and correct if necessary.

Answer: Please keep tables 1a and 1b separate, also keep the footnotes below each corresponding table (as submitted in the original document).

Query: Please check the edits made in Tables $1 \mathrm{a}$ and $\mathrm{b}$ and correct if necessary.

Answer: Please keep tables $1 \mathrm{a}$ and $1 \mathrm{~b}$ separate, also keep the footnotes below each corresponding table (as submitted in the original document). 